\title{
CONVERGENCE OF EXPONENTIAL ATTRACTORS FOR A TIME SEMI-DISCRETE REACTION-DIFFUSION EQUATION
}

\author{
MORGAN PIERRE
}

\begin{abstract}
We consider a time semi-discretization of a generalized Allen-Cahn equation with time-step parameter $\tau$. For every $\tau$, we build an exponential attractor $\mathcal{M}_{\tau}$ of the discrete-in-time dynamical system. We prove that $\mathcal{M}_{\tau}$ converges to an exponential attractor $\mathcal{M}_{0}$ of the continuous-in-time dynamical system for the symmetric Hausdorff distance as $\tau$ tends to 0 . We also provide an explicit estimate of this distance and we prove that the fractal dimension of $\mathcal{M}_{\tau}$ is bounded by a constant independent of $\tau$. Our construction is based on the result of Efendiev, Miranville and Zelik concerning the continuity of exponential attractors under perturbation of the underlying semi-group. Their result has been applied in many situations, but here, for the first time, the perturbation is a discretization. Our method is applicable to a large class of dissipative problems.
\end{abstract}

Keywords: Allen-Cahn equation, backward Euler scheme, global attractor, exponential attractor.

\section{INTRODUCTION}

Understanding and predicting the asymptotic behaviour of systems arising from mechanics and physics is a fundamental issue. A key concept in the study of dissipative systems is the global attractor, a compact invariant set which attracts uniformly the bounded sets of the phase space (see $[3,19,21,25,30]$ for reviews on this subject).

One major drawback of the global attractor is that the rate of attraction of the trajectories may be small, and consequently, it may be sensible to perturbations. In fact, global attractors are generally upper semi-continuous with respect to perturbations, but the lower semi-continuity property can be proved only in some particular cases (see e.g. $[3,24,25,30])$. This includes of course perturbations which are obtained by time and/or space discretization of the governing equations [7, 29, 31, 32].

The notion of exponential attractor has been proposed in [8]: it is a compact positively invariant set which contains the global attractor, has finite fractal dimension and attracts exponentially the trajectories. Compared with the global attractor, an exponential attractor is expected to be more robust to perturbations; it can also capture important transient behaviours. We note however that, contrary to the global attractor, an exponential attractor is not necessarily unique, so that its construction relies upon an algorithm.

The continuity of exponential attractors was shown in [8] for classical Galerkin approximations, but only up to a time shift (see also [14, 16]). In [11], Efendiev, Miranville and Zelik proposed a construction of exponential attractors where the continuity holds without time shift (see also [12]). Moreover, the symmetric Hausdorff distance between the perturbed attractor and the unperturbed attractor is estimated. Their construction, which is based on a uniform "smoothing property" and an appropriate error estimate, is valid in Banach spaces; it has been adapted 
to many situations, including singular perturbations (see $[15,17,24]$ and references therein). But, up to now, perturbations due to time or space discretization have not been considered (see however [1] for related robustness results for Galerkin approximations)

The purpose of this paper is to address the case of a time discretization on a model problem. The natural perturbation parameter is the time step $\tau>0 ; \tau=0$ corresponds to the continuous-in-time system. For every $\tau \geq 0$ small enough, we obtain an exponential attractor $\mathcal{M}_{\tau}$ for the corresponding system, and we prove that $\mathcal{M}_{\tau}$ converges to $\mathcal{M}_{0}$ for the symmetric Hausdorff distance, with an explicit estimate of this distance. Moreover, the fractal dimension of $\mathcal{M}_{\tau}$ is bounded by a constant, and $\mathcal{M}_{\tau}$ attracts the bounded sets of the phase space, uniformly with respect to $\tau$.

We first state the continuity result in an abstract form, in a Banach setting (Theorem 2.5). The assumptions needed here may seem rather lengthy, but they should be interpreted as a methodology (see Remark 2.6 for details). The essential tool is the construction in [11].

The abstract result is applied to a dissipative reaction-diffusion equation with a polynomial nonlinearity on a bounded domain (see (3.1)). This example includes the famous Allen-Cahn [2] in any space dimension, also known as the Chafee-Infante equation [5]. The time semi-discrete problem is provided by the backward Euler scheme (see (4.1)).

For the continuous problem, existence of a global attractor which has finite fractal dimension is well-known (see e.g. [6, 20, 23, 30]), and exponential attractors have been constructed in $[8,9]$. A fully discrete approximation of the problem was considered in [27], where the upper semi-continuity of the global attractor with respect to the discretization parameters was shown in one, two and three space dimension, with growth restriction on the nonlinearity in the latter case.

The proof is organized as follows. The estimates for the continuous problem are derived in Section 3, their discrete counterparts are derived in Section 4, and the error between the continuous and the discrete solution is estimated in Section 5. The main result is summarized in Theorem 6.1. We point out (Corollary 6.2) that this result implies a uniform bound on the dimension of the global attractors, which is generally very difficult to obtain (see e.g. Remark 3 in [13]).

We believe that our abstract result can be applied to a large class of parabolic problems, and for a large choice of time discretizations, possibly with some slight modifications. For instance, the two-dimensional Navier-Stokes equation discretized by the backward Euler scheme could be considered. In this case, the discrete-in-time system should not be defined on the whole Hilbert space, by lack of uniqueness [7], but on a properly chosen ball of radius $R(\tau)$ where $R(\tau) \rightarrow \infty$ as $\tau \rightarrow 0$.

We also expect that our methodology can be generalized to damped wave equations. Finally, we note that it would be very interesting to obtain a result similar to Theorem 6.1 , but for a space discretization instead of a time discretization.

\section{The ABSTRACT SETting}

Throughout Section $2, H$ denotes a Banach space with norm $\|\cdot\|_{H}$. We recall that a continuous-in-time semi-group $\left\{S(t), t \in \mathbb{R}_{+}\right\}$on $H$ is a family of (nonlinear) operators such that $S(t)$ is a continuous operator from $H$ into itself, for all $t \geq 0$, 
with $S(0)=I d$ (identity in $H$ ) and

$$
S(t+s)=S(t) \circ S(s), \quad \forall s, t \geq 0 .
$$

A discrete-in-time semi-group $\{S(t), t \in \mathbb{N}\}$ on $H$ is a family of (nonlinear) operators which satisfy these properties with $\mathbb{R}_{+}(=[0,+\infty))$ replaced by $\mathbb{N}$. A discrete-in-time semi-group will usually be denoted $\left\{S^{n}, n \in \mathbb{N}\right\}$, where $S(=S(1))$ is a continuous (nonlinear) operator from $H$ into itself.

A (continuous or discrete) semi-group $\{S(t), t \geq 0\}$ defines a (continuous or discrete) dynamical system: if $u_{0}$ is the state of the dynamical system at time 0 , then $u(t)=S(t) u_{0}$ is the state at time $t \geq 0$. The term "dynamical system" will sometimes be used instead of "semi-group".

We note that in the definitions above, the Banach space $H$ can be replaced by a metric space (for instance a bounded subset of $H$ ).

Definition 2.1 (Global attractor). Let $\{S(t), t \geq 0\}$ be a continuous or discrete semi-group on $H$. A set $\mathcal{A} \subset H$ is called the global attractor of the dynamical system if the following three conditions are satisfied:

(1) $\mathcal{A}$ is compact in $H$;

(2) $\mathcal{A}$ is invariant, i.e. $S(t) \mathcal{A}=\mathcal{A}$, for all $t \geq 0$;

(3) $\mathcal{A}$ attracts all bounded sets in $H$, i.e., for every bounded set $B$ in $H$,

$$
\lim _{t \rightarrow+\infty} \operatorname{dist}_{H}(S(t) B, \mathcal{A})=0 \text {. }
$$

Here, dist $_{H}$ denotes the non-symmetric Hausdorff semi-distance in $H$ between two subsets, which is defined as

$$
\operatorname{dist}_{H}(A, B)=\sup _{a \in A} \inf _{b \in B}\|a-b\|_{H} .
$$

It is easy to see, thanks to the invariance and the attracting property, that the global attractor, when it exists, is unique [30].

Let $A \subset H$ be a subset of $H$. For $\varepsilon>0$, we denote $N_{\varepsilon}(A, H)$ the minimum number of balls of $H$ of radius $\varepsilon>0$ which are necessary to cover $A$. The fractal dimension of $A$ (see e.g. $[8,30]$ ) is the number

$$
\operatorname{dim}_{F}(A)=\limsup _{\varepsilon \rightarrow 0} \frac{\log \left(N_{\varepsilon}(A, H)\right)}{\log (1 / \varepsilon)} .
$$

Definition 2.2 (Exponential attractor). Let $\{S(t), t \geq 0\}$ be a continuous or discrete semi-group on $H$. A set $\mathcal{M} \subset H$ is an exponential attractor of the dynamical system if the following three conditions are satisfied:

(1) $\mathcal{M}$ is compact in $H$ and has finite fractal dimension;

(2) $\mathcal{M}$ is positively invariant, i.e. $S(t) \mathcal{M} \subset \mathcal{M}$, for all $t \geq 0$;

(3) $\mathcal{M}$ attracts exponentially the bounded subsets of $H$ in the following sense:

$\forall B \subset H$ bounded, $\operatorname{dist}_{H}(S(t) B, \mathcal{M}) \leq \mathcal{Q}\left(\|B\|_{H}\right) e^{-\alpha t}, \quad t \geq 0$,

where the positive constant $\alpha$ and the monotonic function $\mathcal{Q}$ are independent of $B$. Here, $\|B\|_{H}=\sup _{b \in B}\|b\|_{H}$.

The exponential attractor, if it exists, contains the global attractor (actually, the existence of an exponential attractor yields the existence of the global attractor, see $[3,24])$. 
Remark 2.3. If $\mathcal{B}$ is a closed bounded subset of $H$ and if $L$ is a (nonlinear) continuous operator from $\mathcal{B}$ into $\mathcal{B}$, we will say that a set $\mathcal{M}^{d} \subset \mathcal{B}$ is an exponential attractor for (the dynamical system generated by) the iterations of $L$ if (1) $\mathcal{M}^{d}$ is compact and has finite fractal dimension in $H,(2) \mathcal{M}^{d}$ is positively invariant, i.e. $L \mathcal{M}^{d} \subset \mathcal{M}^{d}$, and (3) $\mathcal{M}^{d}$ attracts $\mathcal{B}$ exponentially, i.e.

$$
\operatorname{dist}_{H}\left(L^{n} \mathcal{B}, \mathcal{M}^{d}\right) \leq C e^{-\alpha n}, \quad n \in \mathbb{N},
$$

where $C$ and $\alpha>0$ are independent of $n$.

We first give conditions which ensure the existence of an exponential attractor for a continuous semi-group. These conditions could be weakened [24], but here, we have in mind our perturbation result (see Remark 2.6).

Theorem 2.4. Let $H$ and $V$ be two Banach spaces such that $V$ is compactly embedded into $H$. Let $\left\{S_{0}(t), t \in \mathbb{R}_{+}\right\}$be a continuous semi-group on $H$. Suppose that the following four conditions are satisfied.

H1 (Bounded absorbing set): There exists a bounded set $\mathcal{B}$ in $H$ such that

$$
\forall B \subset H \text { bounded, } \exists t(B) \geq 0,\left(t \geq t(B) \Rightarrow S_{0}(t) B \subset \mathcal{B}\right) .
$$

H2 (Smoothing property): For all $T>0$, for all $u_{1}, u_{2} \in \mathcal{B}$,

$$
\left\|S_{0}(T) u_{1}-S_{0}(T) u_{2}\right\|_{V} \leq c_{1}(T)\left\|u_{1}-u_{2}\right\|_{H},
$$

where the function $c_{1}:(0,+\infty) \rightarrow(0,+\infty)$ is continuous.

H3 (Hölder continuity in time): For all $T>0$, there exist $\beta=\beta(T) \in$ $(0,1]$ and a constant $c_{2}(T)$ such that for all $t_{1}, t_{2} \in[0, T]$, for all $u \in \mathcal{B}$,

$$
\left\|S_{0}\left(t_{1}\right) u-S_{0}\left(t_{2}\right) u\right\|_{H} \leq c_{2}(T)\left|t_{1}-t_{2}\right|^{\beta} .
$$

H4 (Lipschitz continuity on bounded sets): For all $T>0$ and for all $B \subset H$ bounded, there exists $c_{3}(T, B)$ such that for all $t \in[0, T]$, for all $u_{1}, u_{2} \in B$,

$$
\left\|S_{0}(t) u_{1}-S_{0}(t) u_{2}\right\|_{H} \leq c_{3}(T, B)\left\|u_{1}-u_{2}\right\|_{H} .
$$

Then the continuous dynamical system $\left\{S_{0}(t), t \in \mathbb{R}_{+}\right\}$possesses an exponential attractor $\mathcal{M}_{0}$.

Proof. We may assume, without loss of generality, that $\mathcal{B}$ is closed in $H$ (otherwise, replace $\mathcal{B}$ by its closure in $H$ ). We choose $T_{0}>t(\mathcal{B})$ (cf. assumption (H1)) and consider the continuous mapping $L_{0}=S_{0}\left(T_{0}\right): \mathcal{B} \rightarrow \mathcal{B}$. This mapping $L_{0}$ enjoys the smoothing property (assumption (H2) for $T=T_{0}$ ). By Proposition 1 in [10], the dynamical system generated by iterations of $L_{0}$ possesses an exponential attractor (cf. Remark 2.3) which attracts $\mathcal{B}$ exponentially, i.e.

$$
\operatorname{dist}_{H}\left(L_{0}^{n} \mathcal{B}, \mathcal{M}_{0}^{d}\right) \leq C e^{-\alpha n}, n \in \mathbb{N},
$$

where $C$ and $\alpha>0$ only depend on $\mathcal{B}$. Next, we set

$$
\mathcal{M}_{0}=\bigcup_{t \in\left[0, T_{0}\right]} S_{0}(t) \mathcal{M}_{0}^{d} .
$$

Assumptions (H3) and (H4) imply that the function $F(t, u)=S_{0}(t) u$ is $\beta$-Hölder continuous for the time variable and Lipschitz continuous for the phase variable on 
$\left[0, T_{0}\right] \times \mathcal{B}$. Since $\mathcal{M}_{0}=F\left(\left[0, T_{0}\right] \times \mathcal{M}_{0}^{d}\right)$, this shows that $\mathcal{M}_{0}$ is compact and has finite fractal dimension, with

$$
\operatorname{dim}_{F}\left(\mathcal{M}_{0}\right) \leq \frac{1}{\beta}+\operatorname{dim}_{F}\left(\mathcal{M}_{0}^{d}\right)
$$

A standard argument [8] shows that $\mathcal{M}_{0}$ is positively invariant, i.e. $S_{0}(t) \mathcal{M}_{0} \subset \mathcal{M}_{0}$ for all $t \geq 0$, and that $\mathcal{M}_{0}$ attracts $\mathcal{B}$ exponentially, i.e.

$$
\operatorname{dist}_{H}\left(S_{0}(t) \mathcal{B}, \mathcal{M}_{0}\right) \leq C^{\prime} e^{-\alpha^{\prime} t}
$$

where we can choose $C^{\prime}=c_{3}\left(T_{0}, \mathcal{B}\right) C e^{\alpha}$ and $\alpha^{\prime}=\alpha / T_{0}$.

For $R>0$, consider now the closed ball $B_{R}=\left\{u \in H:\|u\|_{H} \leq R\right\}$. Since $\mathcal{B}$ is an absorbing set (assumption (H1)), there exists $t_{R}=t\left(B_{R}\right)$ such that $t \geq t_{R}$ implies $S_{0}(t) B_{R} \subset \mathcal{B}$. Thus, for $t \geq t_{R}$, we have

$$
\begin{aligned}
\operatorname{dist}_{H}\left(S_{0}(t) B_{R}, \mathcal{M}_{0}\right) & =\operatorname{dist}_{H}\left(S_{0}\left(t-t_{R}\right) S_{0}\left(t_{R}\right) B_{R}, \mathcal{M}_{0}\right) \\
& \leq C^{\prime} e^{-\alpha^{\prime}\left(t-t_{R}\right)}
\end{aligned}
$$

For $t \in\left[0, t_{R}\right]$, we have

$$
\operatorname{dist}_{H}\left(S_{0}(t) B_{R}, \mathcal{M}_{0}\right) \leq \operatorname{dist}_{H}\left(\bigcup_{s \in\left[0, t_{R}\right]} S_{0}(s) B_{R}, \mathcal{M}_{0}\right)=: Q_{R}
$$

with $Q_{R}<+\infty$ because the set $\bigcup_{s \in\left[0, t_{R}\right]} S_{0}(s) B_{R}$ is bounded, as well as $\mathcal{M}_{0}$. Indeed, using (H3) and (H4), we see that if $s \in\left[0, t_{R}\right]$ and $u \in B_{R}$, by choosing $u_{1} \in \mathcal{B}$, we have

$$
\begin{aligned}
\left\|S_{0}(s) u\right\|_{H} & \leq\left\|S_{0}(s) u-S_{0}(s) u_{1}\right\|_{H}+\left\|S_{0}(s) u_{1}-S_{0}(0) u_{1}\right\|_{H}+\left\|u_{1}\right\|_{H} \\
& \leq c_{3}\left(t_{R}, B_{R} \cup \mathcal{B}\right)\left(R+\|\mathcal{B}\|_{H}\right)+c_{2}\left(t_{R}\right) t_{R}^{\beta}+\|\mathcal{B}\|_{H} .
\end{aligned}
$$

By setting $\mathcal{Q}(R)=e^{\alpha^{\prime} t_{R}} \max \left(C^{\prime}, Q_{R}\right)$, from (2.5) and (2.6), we deduce

$$
\operatorname{dist}_{H}\left(S_{0}(t) B_{R}, \mathcal{M}_{0}\right) \leq \mathcal{Q}(R) e^{-\alpha^{\prime} t}, \quad t \geq 0,
$$

where $\alpha^{\prime}>0$ and the function $\mathcal{Q}$ are independent of $R$. We note that $\mathcal{Q}$ can easily be changed into a monotonic function by using that $B_{R} \subset B_{R^{\prime}}$ if $R<R^{\prime}$. This finishes the proof.

We now state our perturbation result in a Banach setting. For a possible comparison with concrete situations, we note that the time $t$ and the time step $\tau$ may have a unit, but the elements of $H$ and the norms in $H$ and $V$ are dimensionless.

Theorem 2.5. Let $H$ and $V$ be two Banach spaces such that $V$ is compactly embedded into $H$. Let $\left\{S_{0}(t), t \in \mathbb{R}_{+}\right\}$be a continuous semi-group on $H$ which satisfies assumptions (H1)-(H4) from Theorem 2.4, and let $\left\{S_{\tau}^{n}, n \in \mathbb{N}\right\}, \tau \in\left(0, \tau_{0}\right]\left(\tau_{0}>0\right)$ be a family of discrete semi-groups on $H$. Suppose that the following five additional conditions are satisfied.

H5 (Bounded absorbing set independent of $\tau$ ): The bounded set $\mathcal{B} \subset H$ from assumption (H1) can be chosen such that, for all $B \subset H$ bounded, there is a time $t(B) \geq 0$ such that, for all $\tau \in\left(0, \tau_{0}\right]$ and $n \in \mathbb{N}$ which satisfy $n \tau \geq t(B)$, we have $S_{\tau}^{n} B \subset \mathcal{B}$. 
H6 (Smoothing property, uniform with $\tau$ ): For all $T \geq \tau_{0}$, there exist $\bar{\tau}_{0}(T) \in\left(0, \tau_{0}\right]$ and a constant $c_{4}(T)$ such that for all $\tau \in\left(0, \bar{\tau}_{0}(T)\right]$, for all $u_{1}, u_{2} \in \mathcal{B}$,

$$
\left\|S_{\tau}^{[T / \tau]} u_{1}-S_{\tau}^{[T / \tau]} u_{2}\right\|_{V} \leq c_{4}(T)\left\|u_{1}-u_{2}\right\|_{H} .
$$

Here, $[\cdot]$ is the floor function, i.e. for every real number $x,[x]$ is the largest integer less than or equal to $x$.

H7 (Finite time uniform error estimate): For all $T>0$, there exist $\gamma=$ $\gamma(T) \in(0,1]$ and a constant $c_{5}(T)$ such that, for all $\tau \in\left(0, \tau_{0}\right]$,

$$
\sup _{u \in \mathcal{B}, 0 \leq n \tau \leq T}\left\|S_{\tau}^{n} u-S_{0}(n \tau) u\right\|_{H} \leq c_{5}(T) \tau^{\gamma} .
$$

H8 (Lipschitz continuity on bounded sets, uniform with $\tau$ ): For every time $T>0$ and for all $B \subset H$ bounded, there exists $c_{6}(T, B)$ such that for all $\tau \in\left(0, \tau_{0}\right]$, for all $0 \leq n \tau \leq T$, for all $u_{1}, u_{2} \in B$,

$$
\left\|S_{\tau}^{n} u_{1}-S_{\tau}^{n} u_{2}\right\|_{H} \leq c_{6}(T, B)\left\|u_{1}-u_{2}\right\|_{H} .
$$

H9 (Bound on bounded sets, uniform with $\tau$ ): For all $T>0$ and for all $B \subset H$ bounded, there exists $c_{7}(T, B)$ such that for all $\tau \in\left(0, \tau_{0}\right]$, for all $0 \leq n \tau \leq T$, for all $u \in B$,

$$
\left\|S_{\tau}^{n} u\right\|_{H} \leq c_{7}(T, B) .
$$

Then, for every $\tau \in\left(0, \tau_{0}^{\prime}\right], \tau_{0}^{\prime}>0$ small enough, the discrete dynamical system associated to $\left\{S_{\tau}^{n}, n \in \mathbb{N}\right\}$ possesses an exponential attractor $\mathcal{M}_{\tau}$ on $H$, and the continuous dynamical system $\left\{S_{0}(t), t \in \mathbb{R}_{+}\right\}$possesses an exponential attractor $\mathcal{M}_{0}$ such that:

(1) the fractal dimension of $\mathcal{M}_{\tau}$ is bounded, uniformly with respect to $\tau \in\left[0, \tau_{0}^{\prime}\right]$,

$$
\operatorname{dim}_{F} \mathcal{M}_{\tau} \leq c_{8},
$$

where $c_{8}$ is independent of $\tau$;

(2) $\mathcal{M}_{\tau}$ attracts the bounded sets of $H$, uniformly with respect to $\tau \in\left(0, \tau_{0}^{\prime}\right]$, i.e.

$\forall \tau \in\left(0, \tau_{0}^{\prime}\right], \forall B \subset H$ bounded, $\operatorname{dist}_{H}\left(S_{\tau}^{n} B, \mathcal{M}_{\tau}\right) \leq \mathcal{Q}\left(\|B\|_{H}\right) e^{-c_{9} n \tau}, n \in \mathbb{N}$,

where the constant $c_{9}$ and the monotonic function $\mathcal{Q}$ are independent of $\tau$;

(3) the family $\left\{\mathcal{M}_{\tau}, \tau \in\left[0, \tau_{0}^{\prime}\right]\right\}$ is continuous at 0 ,

$$
\operatorname{dist}_{\text {sym }}\left(\mathcal{M}_{\tau}, \mathcal{M}_{0}\right) \leq c_{10} \tau^{c_{11}}
$$

where $c_{10}$ and $c_{11} \in(0,1)$ are independent of $\tau$ and dist sym $_{\text {denotes the }}$ symmetric Hausdorff distance between sets, defined by

$$
\operatorname{dist}_{\text {sym }}(A, B):=\max \left(\operatorname{dist}_{H}(A, B), \operatorname{dist}_{H}(B, A)\right) .
$$

Remark 2.6. Assumptions (H5), (H6) and (H8) are the discrete counterparts of assumptions (H1), (H2) and (H4) respectively (uniformly with respect to $\tau$ ). Assumption (H9) is a weak discrete version of (H3). Assumption (H7) gives the relation between the continuous and the discrete semi-groups. We stress that the bounded set $\mathcal{B}$ is the same in (H1)-(H3) and (H5)-(H7). 
Remark 2.7. 1. The constants $c_{i}, i=8, \ldots, 11$ can be computed explicitly in terms of the physical parameters of the problem in concrete situations. In particular, from $(2.4),(2.10),(2.13)$, and $[10,11]$ we find that, for all $\tau \in\left[0, \tau_{0}^{\prime}\right]$,

$$
\operatorname{dim}_{F}\left(\mathcal{M}_{\tau}\right) \leq \log _{2}\left[N_{1 /\left(4 c_{4}^{\prime}\right)}(B(0,1 ; V), H)\right]+1 / \beta,
$$

where $N_{\varepsilon}(B(0,1 ; V), H)$ is the number of ball of radius $\varepsilon$ in $H$ which are necessary to cover the unit ball centered at 0 in $V$.

2. The continuity holds at $\tau=0$ only.

Proof. As previously, we may assume that $\mathcal{B}$ is closed in $H$. We use assumption (H5) with $B=\mathcal{B}$, and we set $T_{0}=t(\mathcal{B})+\tau_{0}$. In particular, $S_{0}\left(T_{0}\right) \mathcal{B} \subset \mathcal{B}$. Moreover, for all $\tau \in\left(0, \tau_{0}\right]$, we have $\left[T_{0} / \tau\right] \tau \geq t(\mathcal{B})$, and so $S_{\tau}^{\left[T_{0} / \tau\right]} \mathcal{B} \subset \mathcal{B}$.

Let $\beta \in(0,1)$ be such that $S_{0}$ is $\beta$-Hölder continuous in time on $\left[0, T_{0}\right] \times \mathcal{B}$ (assumption (H3)). We may assume that assumption (H7) holds on $\left[0, T_{0}\right]$ with $\gamma \leq \beta$ (otherwise replace $\gamma$ by $\beta$ ). We define $\tau_{0}^{\prime}=\bar{\tau}_{0}\left(T_{0}\right) \in\left(0, \tau_{0}\right]$ (cf. (H6)). For $\tau \in\left(0, \tau_{0}^{\prime}\right]$, we set $\varepsilon=\varepsilon(\tau)=\left(\tau / \tau_{0}^{\prime}\right)^{\gamma}$ (or, equivalently, $\tau=\tau(\varepsilon)=\varepsilon^{1 / \gamma} \tau_{0}^{\prime}$ ), and we denote $L_{\varepsilon}=S_{\tau}^{\left[T_{0} / \tau\right]}$. We also set $L_{0}=S_{0}\left(T_{0}\right)$. Note that $\varepsilon$, which is a renormalization of $\tau$, belongs to $[0,1]$. By assumptions (H2) and (H6), the family of operators $L_{\varepsilon}: \mathcal{B} \rightarrow \mathcal{B}$ satisfies a uniform smoothing property, i.e., for all $\varepsilon \in[0,1]$, for all $u_{1}, u_{2} \in \mathcal{B}$,

$$
\left\|L_{\varepsilon} u_{1}-L_{\varepsilon} u_{2}\right\|_{V} \leq c_{4}^{\prime}\left\|u_{1}-u_{2}\right\|_{H},
$$

where $c_{4}^{\prime}=\max \left\{c_{1}\left(T_{0}\right), c_{4}\left(T_{0}\right)\right\}$ is independent of $\varepsilon$.

Let $\varepsilon \in(0,1]$. For all $u \in \mathcal{B}$, we have, using the triangle inequality, assumptions (H7) and (H3),

$$
\begin{aligned}
\left\|L_{\varepsilon} u-L_{0} u\right\|_{H} & \leq\left\|S_{\tau}^{\left[T_{0} / \tau\right]} u-S_{0}\left(\left[T_{0} / \tau\right] \tau\right) u\right\|_{H}+\left\|S_{0}\left(\left[T_{0} / \tau\right] \tau\right) u-S_{0}\left(T_{0}\right) u\right\|_{H} \\
& \leq c_{5}\left(T_{0}\right) \tau^{\gamma}+c_{2}\left(T_{0}\right) \tau^{\beta},
\end{aligned}
$$

and so $\left\|L_{\varepsilon} u-L_{0} u\right\|_{H} \leq c_{5}^{\prime} \varepsilon$ where $c_{5}^{\prime}=c_{5}\left(T_{0}\right) \tau_{0}^{\prime \gamma}+c_{2}\left(T_{0}\right) \tau_{0}^{\prime \beta}$ is independent of $\varepsilon$. Assumption (H8) shows that for all $u_{1}, u_{2} \in \mathcal{B}$,

$$
\left\|L_{\varepsilon} u_{1}-L_{\varepsilon} u_{2}\right\|_{H} \leq c_{6}\left(T_{0}, \mathcal{B}\right)\left\|u_{1}-u_{2}\right\|_{H} .
$$

By induction, we find that for every integer $i \geq 1$, for all $u \in \mathcal{B}$,

$$
\left\|L_{\varepsilon}^{i} u-L_{0}^{i} u\right\|_{H} \leq\left(\sum_{j=0}^{i-1} c_{6}\left(T_{0}, \mathcal{B}\right)^{j}\right) c_{5}^{\prime} \varepsilon .
$$

Indeed, we have seen that the estimate (2.11) is satisfied for $i=1$. Assume that it is satisfied for $i \geq 1$. Then

$$
\begin{aligned}
\left\|L_{\varepsilon}^{i+1} u-L_{0}^{i+1} u\right\|_{H} & \leq\left\|L_{\varepsilon}\left(L_{\varepsilon}^{i} u\right)-L_{\varepsilon}\left(L_{0}^{i} u\right)\right\|_{H}+\left\|L_{\varepsilon}\left(L_{0}^{i} u\right)-L_{0}\left(L_{0}^{i} u\right)\right\|_{H} \\
& \leq c_{6}\left(T_{0}, \mathcal{B}\right)\left\|L_{\varepsilon}^{i} u-L_{0}^{i} u\right\|_{H}+c_{5}^{\prime} \varepsilon,
\end{aligned}
$$

and using the induction assumption (2.11) at step $i$, we find that (2.11) is satisfied at step $i+1$. Estimate (2.11) implies that, for some constant $C=C\left(c_{5}^{\prime}, c_{6}\left(T_{0}, \mathcal{B}\right)\right)$ independent of $\varepsilon$, we have, for all $\varepsilon \in[0,1]$, for all $i \in \mathbb{N}$, for all $u \in \mathcal{B}$,

$$
\left\|L_{\varepsilon}^{i} u-L_{0}^{i} u\right\|_{H} \leq C^{i} \varepsilon \text {. }
$$

We may therefore apply the abstract result concerning the existence and convergence of exponential attractors, Theorem 4.4 in [11]. Namely, for every $\varepsilon \in[0,1]$, there 
exists a set $\mathcal{M}_{\varepsilon}^{d} \subset \mathcal{B}$ which is an attractor for the iterations of $L_{\varepsilon}$ (cf. Remark 2.3). Moreover,

(1) the fractal dimension of $\mathcal{M}_{\varepsilon}^{d}$ is bounded, uniformly with respect to $\varepsilon$,

$$
\operatorname{dim}_{F} \mathcal{M}_{\varepsilon}^{d} \leq C_{1},
$$

where $C_{1}$ is independent of $\varepsilon$;

(2) $\mathcal{M}_{\varepsilon}^{d}$ attracts $\mathcal{B}$ uniformly with respect to $\varepsilon$,

$$
\operatorname{dist}_{H}\left(L_{\varepsilon}^{k} \mathcal{B}, \mathcal{M}_{\varepsilon}^{d}\right) \leq C_{2} e^{-C_{3} k}, \quad C_{2}>0, k \in \mathbb{N},
$$

where $C_{2}$ and $C_{3}$ are independent of $\varepsilon$;

(3) the family $\left\{\mathcal{M}_{\varepsilon}^{d}, \varepsilon \in[0,1]\right\}$ is continuous at 0 ,

$$
\operatorname{dist}_{\text {sym }}\left(\mathcal{M}_{\varepsilon}^{d}, \mathcal{M}_{0}^{d}\right) \leq C_{4} \varepsilon^{C_{5}}
$$

where $C_{4}$ and $C_{5} \in(0,1)$ are independent of $\varepsilon$.

Now, for $\tau \in\left(0, \tau_{0}^{\prime}\right]$, we set

$$
\mathcal{M}_{\tau}=\bigcup_{0 \leq n \tau \leq T_{0}} S_{\tau}^{n} \mathcal{M}_{\varepsilon(\tau)}^{d}
$$

The attractor $\mathcal{M}_{0}$ is defined as previously by (2.3) (with $T_{0}$ and $\mathcal{M}_{0}^{d}$ as above). By arguing as in the continuous case (Theorem 2.4), we see that for every $\tau \in\left(0, \tau_{0}^{\prime}\right]$, $\mathcal{M}_{\tau}$ is an exponential attractor for the semi-group $\left\{S_{\tau}^{n}: n \in \mathbb{N}\right\}$. Indeed, for all $n, S_{\tau}^{n}$ is Lipschitz continuous on $\mathcal{B}$ (assumption (H8)), so $\mathcal{M}_{\tau}$ is compact and its fractal dimension satisfies

$$
\operatorname{dim}_{F}\left(\mathcal{M}_{\tau}\right) \leq \operatorname{dim}_{F}\left(\mathcal{M}_{\varepsilon(\tau)}^{d}\right) \leq C_{1} .
$$

Moreover, using $L_{\varepsilon} \mathcal{M}_{\varepsilon}^{d}=S_{\tau}^{\left[T_{0} / \tau\right]} \mathcal{M}_{\varepsilon(\tau)}^{d} \subset \mathcal{M}_{\varepsilon(\tau)}^{d}$, we see that

$$
S_{\tau} \mathcal{M}_{\tau}=\bigcup_{1 \leq n \leq\left[T_{0} / \tau\right]} S_{\tau}^{n} \mathcal{M}_{\varepsilon(\tau)}^{d} \cup S_{\tau}^{\left[T_{0} / \tau\right]+1} \mathcal{M}_{\varepsilon(\tau)}^{d} \subset \mathcal{M}_{\tau} .
$$

Let us show that $\mathcal{M}_{\tau}$ attracts $\mathcal{B}$. For $n \in \mathbb{N}$, we write $n=k\left[T_{0} / \tau\right]+r$ with $k \in \mathbb{N}$ and $r \in\left\{0, \ldots,\left[T_{0} / \tau\right]-1\right\}$. We have

$$
\operatorname{dist}_{H}\left(S_{\tau}^{n} \mathcal{B}, \mathcal{M}_{\tau}\right)=\operatorname{dist}_{H}\left(S_{\tau}^{r} L_{\varepsilon(\tau)}^{k} \mathcal{B}, \mathcal{M}_{\tau}\right) \leq \operatorname{dist}_{H}\left(S_{\tau}^{r} L_{\varepsilon(\tau)}^{k} \mathcal{B}, S_{\tau}^{r} \mathcal{M}_{\varepsilon(\tau)}^{d}\right) .
$$

Next, we use that $S_{\tau}^{r}$ is Lipschitz continuous on $\mathcal{B}$ (cf. (2.7)), and we obtain

$$
\operatorname{dist}_{H}\left(S_{\tau}^{r} L_{\varepsilon(\tau)}^{k} \mathcal{B}, S_{\tau}^{r} \mathcal{M}_{\varepsilon(\tau)}^{d}\right) \leq c_{6}\left(T_{0}, \mathcal{B}\right) \operatorname{dist}_{H}\left(L_{\varepsilon(\tau)}^{k} \mathcal{B}, \mathcal{M}_{\varepsilon(\tau)}^{d}\right) \leq c_{6}\left(T_{0}, \mathcal{B}\right) C_{2} e^{-C_{3} k} .
$$

Using $k>n /\left[T_{0} / \tau\right]-1 \geq n \tau / T_{0}-1$, we note that $e^{-C_{3} k} \leq e^{C_{3}} e^{-C_{3} n \tau / T_{0}}$, and we obtain

$$
\operatorname{dist}_{H}\left(S_{\tau}^{n} \mathcal{B}, \mathcal{M}_{\tau}\right) \leq C_{2}^{\prime} e^{-C_{3}^{\prime} n \tau}
$$

where $C_{2}^{\prime}=c_{6}\left(T_{0}, \mathcal{B}\right) C_{2} e^{C_{3}}$ and $C_{3}^{\prime}=C_{3} / T_{0}$ do not depend on $\tau$.

For $R>0$, we consider now the closed ball $B_{R}=\left\{u \in H:\|u\|_{H} \leq R\right\}$. Since $\mathcal{B}$ is an absorbing set (assumption (H5)), there exists $t_{R}=t\left(B_{R}\right) \geq \tau_{0}^{\prime}$ such that for all $\tau \in\left(0, \tau_{0}^{\prime}\right], n \tau \geq t_{R}-\tau_{0}^{\prime}$ implies $S_{\tau}^{n} B_{R} \subset \mathcal{B}$. Let $\tau \in\left(0, \tau_{0}^{\prime}\right]$. Then, for $n \geq\left[t_{R} / \tau\right]$, since $S_{\tau}^{\left[t_{R} / \tau\right]} B_{R} \subset \mathcal{B}$, we can write

$$
\begin{aligned}
\operatorname{dist}_{H}\left(S_{\tau}^{n} B_{R}, \mathcal{M}_{\tau}\right) & =\operatorname{dist}_{H}\left(S_{\tau}^{n-\left[t_{R} / \tau\right]} S^{\left[t_{R} / \tau\right]} B_{R}, \mathcal{M}_{\tau}\right) \\
& \leq C_{2}^{\prime} e^{-C_{3}^{\prime}\left(n-\left[t_{R} / \tau\right]\right) \tau}
\end{aligned}
$$


Let now $n \in\left\{0, \ldots,\left[t_{R} / \tau\right]\right\}$. Using $(2.8)$, we see that for all $u \in B_{R}$,

$$
\left\|S_{\tau}^{n} u\right\|_{H} \leq c_{7}\left(t_{R}, B_{R}\right) .
$$

which shows that $S_{\tau}^{n} B_{R} \subset B_{g(R)}$ where $g(R)=c_{7}\left(t_{R}, B_{R}\right)$ is independent of $\tau$. Using also $\mathcal{M}_{\tau} \supset \mathcal{M}_{\varepsilon(\tau)}^{d}$, we see that

$$
\operatorname{dist}_{H}\left(S_{\tau}^{n} B_{R}, \mathcal{M}_{\tau}\right) \leq \operatorname{dist}_{H}\left(B_{g(R)}, \mathcal{M}_{\varepsilon(\tau)}^{d}\right) .
$$

Since $\mathcal{M}_{\varepsilon(\tau)} \subset \mathcal{B}$, by the triangle inequality, we have

$$
\operatorname{dist}_{H}\left(B_{g(R)}, \mathcal{M}_{\varepsilon(\tau)}^{d}\right) \leq g(R)+\|\mathcal{B}\|_{H}=: Q_{R} .
$$

By setting $\mathcal{Q}(R)=e^{C_{3}^{\prime} t_{R}} \max \left\{C_{2}^{\prime}, Q_{R}\right\}$, from (2.14)-(2.16), we deduce

$$
\operatorname{dist}_{H}\left(S_{\tau}^{n} B_{R}, \mathcal{M}_{\tau}\right) \leq \mathcal{Q}(R) e^{-C_{3}^{\prime} n \tau}, \quad n \in \mathbb{N},
$$

where $C_{3}^{\prime}$ and the function $\mathcal{Q}$ are independent of $\tau$. The function $\mathcal{Q}$ can easily be changed into a monotonic function by using that $B_{R} \subset B_{R^{\prime}}$ if $R<R^{\prime}$. This yields conclusion (2) of Theorem 2.5.

It remains to prove conclusion (3). Let $\tau \in\left(0, \tau_{0}^{\prime}\right]$. Using the definitions of $\mathcal{M}_{0}$, $\mathcal{M}_{\tau}$ and the triangle inequality, we see that

$$
\operatorname{dist}_{\text {sym }}\left(\mathcal{M}_{\tau}, \mathcal{M}_{0}\right) \leq h_{1}(\tau)+h_{2}(\tau)+h_{3}(\tau),
$$

where

$$
\begin{aligned}
& h_{1}(\tau)=\operatorname{dist}_{\text {sym }}\left(\bigcup_{0 \leq n \tau \leq T_{0}} S_{\tau}^{n} \mathcal{M}_{\varepsilon(\tau)}^{d}, \bigcup_{0 \leq n \tau \leq T_{0}} S_{0}(n \tau) \mathcal{M}_{\varepsilon(\tau)}^{d}\right), \\
& h_{2}(\tau)=\operatorname{dist}_{\text {sym }}\left(\bigcup_{0 \leq n \tau \leq T_{0}} S_{0}(n \tau) \mathcal{M}_{\varepsilon(\tau)}^{d}, \bigcup_{0 \leq n \tau \leq T_{0}} S_{0}(n \tau) \mathcal{M}_{0}^{d}\right), \\
& h_{3}(\tau)=\operatorname{dist}_{\text {sym }}\left(\bigcup_{0 \leq n \tau \leq T_{0}} S_{0}(n \tau) \mathcal{M}_{0}^{d}, \bigcup_{0 \leq t \leq T_{0}} S_{0}(t) \mathcal{M}_{0}^{d}\right) .
\end{aligned}
$$

By assumption (H7), we have $h_{1}(\tau) \leq c_{5}\left(T_{0}\right) \tau^{\gamma}$. Using that $S_{0}(n \tau)$ is Lipschitz continuous on $\mathcal{B}$ (assumption (H3)), we have

$$
h_{2}(\tau) \leq c_{3}\left(T_{0}, \mathcal{B}\right) \operatorname{dist}_{\text {sym }}\left(\mathcal{M}_{\varepsilon(\tau)}^{d}, \mathcal{M}_{0}^{d}\right) \leq c_{3}\left(T_{0}, \mathcal{B}\right) C_{4}\left(\tau / \tau_{0}^{\prime}\right)^{C_{5} \gamma} .
$$

Using that $S_{0}$ is $\beta$-Hölder continuous in time (assumption (H3)), we find that

$$
h_{3}(\tau) \leq c_{2}\left(T_{0}\right) \tau^{\beta} .
$$

Summing up, we have proved that

$$
\operatorname{dist}_{\text {sym }}\left(\mathcal{M}_{\tau}, \mathcal{M}_{0}\right) \leq c_{10} \tau^{c_{11}},
$$

where $c_{10}$ and $c_{11}=C_{5} \gamma \in(0,1)$ are independent of $\tau$. This concludes the proof.

\section{The Continuous PRoblem}

3.1. The continuous semi-group $S_{0}$. We consider the following reaction-diffusion equation

$$
\partial_{t} u-d \Delta u+g(u)=0 \quad \text { in } \Omega \times \mathbb{R}_{+},
$$


subject to homogeneous Dirichlet boundary conditions. Here, $\Omega$ is an open bounded subset of $\mathbb{R}^{I}(I \geq 1)$ with sufficiently smooth boundary, $d>0$ is given, and $g$ is a polynomial of odd degree with a positive leading coefficient,

$$
g(s)=\sum_{j=0}^{2 p-1} b_{j} s^{j}, \quad b_{2 p-1}>0, p \geq 1 .
$$

Note that equation (3.1) is linear if and only if $p=1$. When $g(s)=s^{3}-s$ (in which case $p=2$ ), equation (3.1) is known as the Allen-Cahn equation.

We supplement (3.1) with an initial condition

$$
u(0)=u_{0} .
$$

We set $H=L^{2}(\Omega)$ with norm $|\cdot|_{H}$ and scalar product $(\cdot, \cdot)_{H}$. We denote $V=H_{0}^{1}(\Omega)$ with norm $\|\cdot\|_{V}=|\nabla \cdot|_{H}$. For an nonempty interval $J$ of $\mathbb{R}$ and for a Banach space $E$, we denote $C^{0}(J ; E)$ the space of functions which are continuous on $J$ with values in $E$; for $q \geq 1, L^{q}(J ; E)$ is the usual Banach space of (classes of) functions endowed with the norm $v \mapsto\left(\int_{J}\|v(t)\|_{E}^{q} d t\right)^{1 / q}$. The norm in $L^{q}(\Omega)$ is denoted $\|\cdot\|_{L^{q}}$.

The following existence and uniqueness result is well-known (see e.g. $[22,30]$ ).

Theorem 3.1. For $u_{0} \in H$, there exists a unique solution $u$ of (3.1)-(3.2) which satisfies $u \in C^{0}\left(\mathbb{R}_{+} ; H\right)$ and $u \in L^{2}(0, T ; V) \cap L^{2 p}\left(0, T ; L^{2 p}(\Omega)\right)$, for all $T>0$. For all $t \geq 0$, the mapping $u_{0} \mapsto u(t)$ is continuous in $H$. If, furthermore, $u_{0} \in V$, then $u$ belongs to $C^{0}([0, T] ; V) \cap L^{2}\left(0, T ; H^{2}(\Omega)\right)$, for all $T>0$.

This theorem is sufficient to define the continuous-in-time semi-group $S_{0}$ :

$$
S_{0}(t): u_{0} \in H \mapsto u(t) \in H .
$$

3.2. Some useful inequalities. First recall the Poincaré inequality: there exists a constant $c_{0}=c_{0}(I, \Omega)$ such that

$$
|w|_{H} \leq c_{0}\|w\|_{V}, \quad \forall w \in V .
$$

More generally, thanks to the Sobolev imbeddings (see e.g. [18]), there is a constant $C_{S}(I, \Omega, q)$ such that

$$
\|w\|_{L^{q}} \leq C_{S}(I, \Omega, q)\|w\|_{V}, \quad \forall w \in V,
$$

for every $q \in[1,+\infty)$ if $I=1$ or $I=2$ and for every $q \in[1,2 I /(I-2)]$ if $I \geq 3$.

In our a priori estimates, we will consider two cases:

Case 1: $I \in\{1,2\}$ (no restriction on $p$ ), or $I=3$ and $p \in\{1,2\}$, or $I \geq 4$ and $p=1$

Case 2: $I=3$ and $p \geq 3$, or $I \geq 4$ and $p \geq 2$.

If Case 1 holds, then we have the continuous imbeddings

$$
V \subset L^{4 p-2}(\Omega) \subset L^{2 p}(\Omega) .
$$

In this case, a bounded absorbing set in $V$ will be sufficient. In Case 2, we will consider a bounded absorbing set in $V \cap L^{q}(\Omega)$ for $q$ large enough, so that we will need estimates both in $V$ and in $L^{q}(\Omega)$. In fact, both cases could be treated with a bounded set in $V \cap L^{\infty}$, by using more fully the regularization properties of the equation. But for other problems, $L^{\infty}$ estimates may be difficult to obtain, so that we prefer this approach here. We note that Case 1 includes the Allen-Cahn equation in space dimension 1, 2 and $3\left(g(s)=s^{3}-s\right.$ with $\left.p=2\right)$. 
Next, we collect a few inequalities related to $g$. Since $\sum_{j=1}^{2 p-2} j b_{j} s^{j-1}$ is a polynomial of degree less than $s^{2 p-2}$, there exists a constant $c_{1}^{\prime}>0$ such that

$$
\left|\sum_{j=1}^{2 p-2} j b_{j} s^{j-1}\right| \leq \frac{1}{2}(2 p-1) b_{2 p-1} s^{2 p-2}+c_{1}^{\prime}, \quad \forall s \in \mathbb{R} .
$$

Thus, $g^{\prime}(s)=\sum_{j=1}^{2 p-1} j b_{j} s^{j-1}$ satisfies

$$
\left|g^{\prime}(s)\right| \leq \frac{3}{2}(2 p-1) b_{2 p-1} s^{2 p-2}+c_{1}^{\prime}, \quad \forall s \in \mathbb{R},
$$

and

$$
\frac{2 p-1}{2} b_{2 p-1} s^{2 p-2}-c_{1}^{\prime} \leq g^{\prime}(s) \leq \frac{3}{2}(2 p-1) b_{2 p-1} s^{2 p-2}+c_{1}^{\prime}, \quad \forall s \in \mathbb{R} .
$$

We note that by the mean value theorem we have, for all $s_{1}, s_{2} \in \mathbb{R}$,

$$
\left(g\left(s_{1}\right)-g\left(s_{2}\right)\right)\left(s_{1}-s_{2}\right)=g^{\prime}\left(\xi_{s_{1}, s_{2}}\right)\left(s_{1}-s_{2}\right)^{2} \geq-c_{1}^{\prime}\left(s_{1}-s_{2}\right)^{2},
$$

for some $\xi_{s_{1}, s_{2}} \in \mathbb{R}$. Let

$$
G(s)=\int_{0}^{s} g(\sigma) d \sigma=\sum_{j=0}^{2 p-1} b_{j} s^{j+1} /(j+1)
$$

denote an anti-derivative of $g$. Using a similar argument, we have

$$
\frac{1}{4 p} b_{2 p-1} s^{2 p}-\hat{c}_{1}^{\prime} \leq G(s) \leq \frac{3}{4 p} b_{2 p-1} s^{2 p}+\hat{c}_{1}^{\prime}, \quad \forall s \in \mathbb{R},
$$

for some constant $\hat{c}_{1}^{\prime}>0$.

By a similar argument, for every $q \geq 2$, there exists a constant $c_{q}^{\prime}>0$ such that

$$
\frac{1}{2} b_{2 p-1}|s|^{2 p+q-2}-c_{q}^{\prime} \leq g(s)|s|^{q-2} s \leq \frac{3}{2} b_{2 p-1}|s|^{2 p+q-2}+c_{q}^{\prime}, \quad \forall s \in \mathbb{R} .
$$

Lemma 3.2. Let $w_{1}, w_{2} \in V$ and $w_{3} \in H$.

If Case 1 holds and $\left\|w_{i}\right\|_{V} \leq R_{1}(i=1,2)$, then

$$
\int_{\Omega}\left|g\left(w_{1}\right)-g\left(w_{2}\right)\right|\left|w_{3}\right| d x \leq h_{1}\left(R_{1}\right)\left\|w_{1}-w_{2}\right\|_{V}\left|w_{3}\right|_{H},
$$

where $h_{1}\left(R_{1}\right)=h_{1}\left(R_{1}, I, \Omega, p, b_{2 p-1}, c_{1}^{\prime}\right)$ is monotonic in $R_{1}$.

If Case 2 holds and $\left\|w_{i}\right\|_{L^{I(2 p-2)}} \leq R_{2}(i=1,2)$, then

$$
\int_{\Omega}\left|g\left(w_{1}\right)-g\left(w_{2}\right)\right|\left|w_{3}\right| d x \leq h_{2}\left(R_{2}\right)|| w_{1}-w_{2} \|_{V}\left|w_{3}\right|_{H},
$$

where $h_{2}\left(R_{2}\right)=h_{2}\left(R_{2}, I, \Omega, p, b_{2 p-1}, c_{1}^{\prime}\right)$ is monotonic in $R_{2}$.

Proof. First note that for all $s_{1}, s_{2} \in \mathbb{R}$, we have

$$
g\left(s_{1}\right)-g\left(s_{2}\right)=\int_{0}^{1} g^{\prime}\left(\sigma s_{1}+(1-\sigma) s_{2}\right)\left(s_{1}-s_{2}\right) d \sigma .
$$

Using (3.5) and the convexity of the function $s \mapsto|s|^{2 p-2}$, we find

$$
\left|g\left(s_{1}\right)-g\left(s_{2}\right)\right| \leq\left[b_{2 p-1}^{\prime}\left(\left|s_{1}\right|^{2 p-2}+\left|s_{2}\right|^{2 p-2}\right)+c_{1}^{\prime}\right]\left|s_{1}-s_{2}\right|, \quad \forall s_{1}, s_{2} \in \mathbb{R},
$$

where $b_{2 p-1}^{\prime}=\frac{3}{4}(2 p-1) b_{2 p-1}$. 
Let $w_{1}, w_{2} \in V$ and $w_{3} \in H$. By (3.13), we have

$$
\int_{\Omega}\left|g\left(w_{1}\right)-g\left(w_{2}\right)\right|\left|w_{3}\right| d x \leq \int_{\Omega}\left[b_{2 p-1}^{\prime}\left(\left|w_{1}\right|^{2 p-2}+\left|w_{2}\right|^{2 p-2}\right)+c_{1}^{\prime}\right]\left|w_{1}-w_{2}\right|\left|w_{3}\right| d x .
$$

Assume first that Case 1 holds and that $\left\|w_{i}\right\|_{V} \leq R_{1}(i=1,2)$. If $p=1$ (the linear case), then we may use the Cauchy-Schwarz inequality and the Poincaré inequality (3.3), and we find (3.11) with $h_{1}=\left(2 b_{2 p-1}^{\prime}+c_{1}^{\prime}\right) c_{0}$ (here, $h_{1}$ does not depend on $R_{1}$ ). If $p \geq 2$, we use that $V \subset L^{4 p-2}(\Omega)$, i.e. (3.4) holds with $q=4 p-2$ and $\hat{C}_{S}=C_{S}(I, \Omega, 4 p-2)$. In (3.14), we use Hölder's inequality with $\left|w_{i}\right|^{2 p-2} \in$ $L^{(4 p-2) /(2 p-2)}(\Omega)(i=1,2), w_{1}-w_{2} \in L^{4 p-2}(\Omega)$ and $w_{3} \in L^{2}(\Omega)$. We obtain (3.11) with

$$
h_{1}\left(R_{1}\right)=\left[2 b_{2 p-1}^{\prime} \hat{C}_{S}^{2 p-2} R_{1}^{2 p-2}+c_{1}^{\prime}|\Omega|^{(2 p-2) /(4 p-2)}\right] \hat{C}_{S} .
$$

Assume now that Case 2 holds and that $\left\|w_{i}\right\|_{L^{I(2 p-2)}} \leq R_{2}(i=1,2)$. We use that $V \subset L^{2^{\star}}(\Omega)$ with $2^{\star}=2 I /(I-2)$, i.e. (3.4) holds with $q=2^{\star}$ and $C_{S}^{\star}=C_{S}\left(I, \Omega, 2^{\star}\right)$. In (3.14), we use Hölder's inequality with $\left|w_{i}\right|^{2 p-2} \in L^{I}(\Omega)(i=1,2), w_{1}-w_{2} \in$ $L^{2^{\star}}(\Omega)$, and $w_{3} \in L^{2}(\Omega)$. We obtain (3.12) with

$$
h_{2}\left(R_{2}\right)=\left[2 b_{2 p-1}^{\prime}\left(C_{S}^{\star}\right)^{2 p-2} R_{2}^{2}+c_{1}^{\prime}|\Omega|^{1 / I}\right] C_{S}^{\star} .
$$

This concludes the proof.

3.3. A priori estimates for the solution. In Section 3.3, $u$ denotes a solution of (3.1)-(3.2). We denote $\rho_{0}^{2}=c_{2}^{\prime \prime} c_{0}^{2} /(2 d), \rho_{0}^{\prime 2}=2 \rho_{0}^{2}$ and, for every $q \geq 2$,

$$
c_{q}^{\prime \prime}=q c_{q}^{\prime}|\Omega| \quad \text { where } \quad|\Omega|=\int_{\Omega} 1 d x .
$$

Propositions 3.3 and 3.4 are proved in [30].

Proposition 3.3 (Absorbing set in $H$ ). If $\left|u_{0}\right|_{H} \leq R$, then

$$
|u(t)|_{H} \leq \rho_{0}^{\prime}, \quad \forall t \geq t_{0}(R),
$$

where

$$
t_{0}(R)=\max \left\{\frac{c_{0}^{2}}{d} \log \left(\frac{R}{\rho_{0}}\right), 0\right\} .
$$

In the remainder of the paper, $r>0$ denotes an arbitrary (but fixed) real number ( $r$ has the same unit as $t$ ).

Proposition 3.4 (Absorbing set in $V$ ). If $\left|u_{0}\right|_{H} \leq R$, then

$$
\|u(t)\|_{V} \leq \rho_{1}, \quad t \geq t_{1}(R),
$$

where

$$
\rho_{1}^{2}=\frac{\kappa}{r} \exp \left(2 c_{1}^{\prime} r\right), \quad \kappa=\frac{1}{2 d}\left(r c_{2}^{\prime \prime}+\rho_{0}^{\prime 2}\right), \quad t_{1}(R)=t_{0}(R)+r .
$$

Lemma 3.5. Let $q \geq 2$. The function $y(t)=\int_{\Omega}|u(t)|^{q} d x$ is locally integrable on $(0,+\infty)$, as well as $d y / d t$. Moreover,

$$
\frac{d}{d t} \int_{\Omega}|u|^{q} d x+\frac{q}{2} b_{2 p-1} \int_{\Omega}|u|^{2 p+q-2} \leq c_{q}^{\prime \prime} \text { on }(0,+\infty) .
$$

In particular, if $u_{0} \in L^{q}(\Omega)$, then for all $t \geq 0$, we have

$$
\int_{\Omega}|u(t)|^{q} d x \leq \int_{\Omega}\left|u_{0}\right|^{q}+c_{q}^{\prime \prime} t .
$$


Proof. By parabolic regularity $[4,26], u \in C^{0}\left((0,+\infty) ; C_{0}(\Omega)\right.$ ) (where $C_{0}(\Omega)$ is the space of continuous functions on $\bar{\Omega}$ which vanish on $\partial \Omega$ ) and $\partial_{t} u, \Delta u$ belong to $C^{0}\left((0,+\infty) ; L^{2}(\Omega)\right)$. In particular, the functions $y$ and $d y / d t$ are locally integrable. We multiply (3.1) by $|u|^{q-2} u$ and integrate over $\Omega$. We obtain, after some simple transformations,

$$
\frac{1}{q} \frac{d}{d t} \int_{\Omega}|u|^{q} d x+(q-1) d \int_{\Omega}|\nabla u|^{2}|u|^{q-2} d x+\int_{\Omega} g(u)|u|^{q-2} u d x=0 .
$$

Using (3.10), (3.15), we obtain (3.19). We infer (3.20) by integration on $[0, t]$.

For all $i \in \mathbb{N}$, we set $a_{i}=i(2 p-2)+2$. We introduce the sequence $\left(\rho_{i}^{\prime}\right)_{i}$ defined recursively for $i \in \mathbb{N}$ by

$$
\rho_{i+1}^{\prime a_{i+1}}=\frac{\kappa_{i}^{\prime}}{r}+r c_{a_{i+1}}^{\prime \prime}, \quad \text { with } \quad \kappa_{i}^{\prime}=\frac{2}{a_{i} b_{2 p-1}}\left(r c_{a_{i}}^{\prime \prime}+\rho_{i}^{\prime a_{i}}\right)
$$

Proposition 3.6 (Absorbing set in $L^{q}$ ). Let $i \in \mathbb{N}$. If $\left|u_{0}\right|_{H} \leq R$, then

$$
\|u(t)\|_{L^{a_{i}}} \leq \rho_{i}^{\prime}, \quad t \geq t_{i}(R)
$$

where $t_{i}(R)=t_{0}(R)+i r$.

Proof. We proceed by induction. We first note that (3.21) is satisfied for $i=0$ by (3.16). Let $i \geq 0$ and assume that (3.21) holds. We first apply (3.19) with $q=a_{i}$ and we integrate on $[t, t+r]$. This yields

$$
\frac{a_{i}}{2} b_{2 p-1} \int_{t}^{t+r}\|u(s)\|_{L^{a_{i+1}}}^{a_{i+1}} d s \leq r c_{a_{i}}^{\prime \prime}+\|u(t)\|_{L^{a_{i}}}^{a_{i}}, \quad \forall t \geq 0 .
$$

Using the induction assumption (3.21), we see that

$$
\int_{t}^{t+r}\|u(s)\|_{L^{a+1}}^{a_{i+1}} d s \leq \kappa_{i}^{\prime}, \quad \forall t \geq t_{i}(R)
$$

Next, we apply (3.19) with $q=a_{i+1}$, and we use the uniform Gronwall lemma [30]. We obtain that (3.21) is satisfied at step $i+1$. By induction, (3.21) is satisfied for every $i \in \mathbb{N}$.

We introduce the monotonic function

$$
Q_{1}(s)=\frac{3 b_{2 p-1}}{2 d p} s^{2 p}+\frac{4 \hat{c}_{1}^{\prime}|\Omega|}{d} .
$$

Lemma 3.7. If $u_{0} \in V \cap L^{2 p}(\Omega)$, then

$$
\|u(t)\|_{V}^{2}+\frac{b_{2 p-1}}{2 d p}\|u(t)\|_{L^{2 p}}^{2 p}+\frac{2}{d} \int_{0}^{t}\left|\partial_{t} u\right|_{H}^{2} d s \leq\left\|u_{0}\right\|_{V}^{2}+Q_{1}\left(\left\|u_{0}\right\|_{L^{2 p}}\right), \quad \forall t \geq 0,
$$

where $Q_{1}$ is defined by (3.23). In particular, for all $t_{1}, t_{2} \geq 0$, we have

$$
\left|u\left(t_{1}\right)-u\left(t_{2}\right)\right|_{H}^{2} \leq Q_{2}\left(\left\|u_{0}\right\|_{V},\left\|u_{0}\right\|_{L^{2 p}}\right)\left|t_{1}-t_{2}\right|
$$

for some function $Q_{2}$ which is monotonic in its arguments.

Proof. We multiply (3.1) by $\partial_{t} u$ and integrate over $\Omega$. We obtain, after integration by parts,

$$
\left|\partial_{t} u\right|_{H}^{2}+\frac{d}{d t}\left(\frac{d}{2}\|u\|_{V}^{2}+\int_{\Omega} G(u) d x\right)=0
$$


Integrating on $[0, t]$ yields

$$
\frac{d}{2}\|u(t)\|_{V}^{2}+\int_{\Omega} G(u(t)) d x+\int_{0}^{t}\left|\partial_{t} u\right|_{H}^{2} d s \leq \frac{d}{2}\left\|u_{0}\right\|_{V}^{2}+\int_{\Omega} G\left(u_{0}\right) d x .
$$

Estimate (3.24) follows from (3.9). Let now $t_{2} \geq t_{1} \geq 0$. Then

$$
\left|u\left(t_{2}\right)-u\left(t_{1}\right)\right|_{H}^{2}=\left|\int_{t_{1}}^{t_{2}} \partial_{t} u(s) d s\right|_{H}^{2} \leq\left|t_{2}-t_{1}\right| \int_{t_{1}}^{t_{2}}\left|\partial_{t} u(s)\right|_{H}^{2} d s,
$$

and (3.25) follows from (3.24) with

$$
Q_{2}\left(\left\|u_{0}\right\|_{V},\left\|u_{0}\right\|_{L^{2 p}}\right)=\frac{d}{2}\left(\left\|u_{0}\right\|_{V}^{2}+Q_{1}\left(\left\|u_{0}\right\|_{L^{2 p}}\right)\right) .
$$

3.4. Estimates for the difference of solutions. In the following lemmas, $u_{1}$ and $u_{2}$ denote two solutions of $(3.1)$, and $v(t)=u_{1}(t)-u_{2}(t)$ is their difference, which satisfies

$$
\partial_{t} v-d \Delta v+g\left(u_{1}\right)-g\left(u_{2}\right)=0 \quad \text { in } \Omega \times \mathbb{R}_{+} .
$$

Lemma 3.8. For all $t \geq 0$,

$$
|v(t)|_{H}^{2}+2 d \int_{0}^{t}\|v\|_{V}^{2} d s \leq|v(0)|_{H}^{2} \exp \left(2 c_{1}^{\prime} t\right) .
$$

Proof. We multiply (3.28) by $2 v$, integrate over $\Omega$, and use (3.7). We obtain

$$
\frac{d}{d t}|v|_{H}^{2}+2 d\|v\|_{V}^{2} \leq 2 c_{1}^{\prime}|v|_{H}^{2}
$$

The classical Gronwall lemma yields (3.29).

We introduce the functions

$$
\tilde{Q}_{1}(s)=\left(s^{2}+Q_{1}\left(C_{S}(I, \Omega, 2 p) s\right)\right)^{1 / 2},
$$

where $C_{S}$ is the Sobolev constant (3.4), $Q_{1}$ is given by (3.23) and

$$
\tilde{h}_{1}\left(R_{1}\right)=\frac{1}{2 d} h_{1}^{2}\left(\tilde{Q}_{1}\left(R_{1}\right)\right), \quad R_{1}>0,
$$

where $h_{1}$ is defined by (3.11).

The following two lemmas show a smoothing property.

Lemma 3.9. Assume that Case 1 holds. If $\left\|u_{i}(0)\right\|_{V} \leq R_{1}(i=1,2)$, then for all $t>0$, we have

$$
\|v(t)\|_{V}^{2} \leq C_{1}\left(t, R_{1}\right)|v(0)|^{2}
$$

where the function $C_{1}:(0,+\infty)^{2} \rightarrow \mathbb{R}_{+}$is continuous.

Proof. Using $V \subset L^{2 p}(\Omega)$, Lemma 3.7 and (3.4) with $q=2 p$, we first note that

$$
\left\|u_{i}(t)\right\|_{V} \leq \tilde{Q}_{1}\left(R_{1}\right), \quad \forall t \geq 0,
$$

for $i=1,2$. Next, we multiply (3.28) by $t \partial_{t} v$ and integrate over $\Omega$. We obtain

$$
t\left|\partial_{t} v\right|_{H}^{2}+\frac{t d}{2} \frac{d}{d t}\|v\|_{V}^{2}=-t \int_{\Omega}\left[g\left(u_{1}\right)-g\left(u_{2}\right)\right] \partial_{t} v d x
$$


Using (3.11) and Young's inequality, we find

$$
\frac{t d}{2} \frac{d}{d t}\left(\|v\|_{V}^{2}\right) \leq \frac{t}{4} h_{1}^{2}\left(\tilde{Q}_{1}\left(R_{1}\right)\right)\|v\|_{V}^{2}, \quad t \geq 0
$$

that is

$$
\frac{d}{d t}\left(t\|v\|_{V}^{2}\right) \leq \tilde{h}_{1}\left(R_{1}\right)\left(t\|v\|_{V}^{2}\right)+\|v\|_{V}^{2}, \quad t \geq 0 .
$$

The classical Gronwall lemma yields

$$
t\|v(t)\|_{V}^{2} \leq \exp \left(\tilde{h}_{1}\left(R_{1}\right) t\right) \int_{0}^{t}\|v(s)\|_{V}^{2} d s, \quad \forall t \geq 0 .
$$

We infer from (3.29) that

$$
t\|v(t)\|_{V}^{2} \leq \frac{1}{2 d} \exp \left(2 c_{1}^{\prime} t\right) \exp \left(\tilde{h}_{1}\left(R_{1}\right) t\right)|v(0)|_{H}^{2}, \quad \forall t \geq 0,
$$

i.e. estimate (3.32) holds.

When Case 2 holds, we use the functions

$$
\tilde{Q}_{2}\left(t, R_{2}\right)=\left(R_{2}^{q}+c_{q}^{\prime \prime} t\right)^{1 / q}, \quad t>0, R_{2}>0
$$

with $q=I(2 p-2)$, and

$$
\tilde{h}_{2}\left(t, R_{2}\right)=\frac{1}{2 d} h_{2}^{2}\left(\tilde{Q}_{2}\left(t, R_{2}\right)\right), \quad t>0, R_{2}>0,
$$

where $h_{2}$ is defined by (3.12).

Lemma 3.10. Assume that Case 2 holds. If $\left\|u_{i}(0)\right\|_{L^{I(2 p-2)}} \leq R_{2}(i=1,2)$, then for all $t>0$, we have

$$
\|v(t)\|_{V}^{2} \leq C_{2}\left(t, R_{2}\right)|v(0)|_{H}^{2},
$$

where the function $C_{2}:(0,+\infty)^{2} \rightarrow \mathbb{R}_{+}$is continuous.

Proof. Let $T>0$. Using (3.20) with $q=I(2 p-2)$, we note that

$$
\left\|u_{i}(t)\right\|_{L^{I(2 p-2)}} \leq \tilde{Q}_{2}\left(T, R_{2}\right), \quad \forall t \in[0, T] .
$$

Arguing as in the proof of Lemma 3.9, and using (3.12), we obtain

$$
\frac{d}{d t}\left(t\|v\|_{V}^{2}\right) \leq \tilde{h}_{2}\left(T, R_{2}\right)\left(t\|v\|_{V}^{2}\right)+\|v\|_{V}^{2}, \quad \forall t \in[0, T] .
$$

The classical Gronwall lemma and (3.29) yield

$$
\left.t\|v(t)\|_{V}^{2} \leq \frac{1}{2 d} \exp \left(2 c_{1}^{\prime} t\right) \exp \tilde{h}_{2}\left(T, R_{2}\right) t\right)|v(0)|_{H}^{2}, \quad \forall t \in[0, T] .
$$

Using this for $t=T$, we obtain (3.36). 


\section{The TIME SEMI-DISCRETE PROBLEM}

4.1. The discrete semi-group. For the time semi-discretization, we apply the backward Euler scheme to (3.1). Throughout this section, $\tau>0$ denotes the time step. The scheme reads: let $u^{0} \in H$ and for $n=0,1,2, \ldots$, let $u^{n+1} \in V \cap L^{2 p}(\Omega)$ solve

$$
\frac{u^{n+1}-u^{n}}{\tau}-d \Delta u^{n+1}+g\left(u^{n+1}\right)=0 .
$$

The following result shows that the discrete semi-group $S_{\tau}^{n} u^{0}=u^{n}$ is well-defined.

Theorem 4.1. Assume that $\tau \leq 1 / c_{1}^{\prime}$. Then for every $u \in H$, there exists a unique $v=v_{\tau, u} \in V \cap L^{2 p}(\Omega)$ such that

$$
\frac{v-u}{\tau}-d \Delta v+g(v)=0 \text { in } V^{\prime}+L^{2 p /(2 p-1)}(\Omega) .
$$

Moreover, the mapping $S_{\tau}: u \mapsto v_{\tau, u}$ is Lipschitz continuous from $H$ into $V$, with

$$
\left\|S_{\tau} u-S_{\tau} \hat{u}\right\|_{V} \leq \frac{c_{0}}{d \tau}|u-\hat{u}|_{H}, \quad \forall u, \hat{u} \in H .
$$

As a consequence, $S_{\tau}$ is Lipschitz continuous from $H$ into $H$, and from $V$ into $V$. We note that the Lipschitz constant blows up as $\tau \rightarrow 0^{+}$.

Proof. Let $u \in H$. We can obtain $v$ by minimizing the function

$$
\mathcal{G}(w)=\frac{|w-u|_{H}^{2}}{2 \tau}+\frac{d}{2}\|w\|_{V}^{2}+\int_{\Omega} G(w)
$$

in the space $V \cap L^{2 p}(\Omega)$. Let now $\hat{u} \in H$, and consider a solution $\hat{v}$ of (4.2) associated to $\hat{u}$. Then the difference $w=v-\hat{v}$ satisfies

$$
\frac{w}{\tau}-d \Delta w+g(v)-g(\hat{v})=\frac{u-\hat{u}}{\tau}
$$

We multiply by $w$, integrate over $\Omega$, use (3.7) and the Cauchy-Schwarz inequality. We obtain

$$
\frac{|w|_{H}^{2}}{\tau}+d\|w\|_{V}^{2}-c_{1}^{\prime}|w|_{H}^{2} \leq \frac{1}{\tau}|u-\hat{u}|_{H}|w|_{H} .
$$

Using $1 / \tau \geq c_{1}^{\prime}$ and the Poincaré inequality (3.3), we obtain

$$
\|w\|_{V} \leq \frac{c_{0}}{d \tau}|u-\hat{u}|_{H} .
$$

Since $w=v-\hat{v}$, this shows that $v$ is unique and that (4.3) holds.

In the remainder of the paper, we will assume that the time step $\tau$ satisfies at least $0<\tau \leq 1 / c_{1}^{\prime}$.

4.2. A priori estimates for the solution, uniform in $\tau$. We use the same notation as in Section 3. Throughout Section 4.2, $\left(u^{n}\right)$ denotes a sequence in $H$ which complies with (4.1). The following well-known identity will prove useful:

$$
(a-b, a)_{H}=\frac{1}{2}\left(|a|_{H}^{2}-|b|_{H}^{2}+|a-b|_{H}^{2}\right), \quad \forall a, b \in H .
$$

Proposition 4.2 (Absorbing set in $H$ ). Assume that $\tau \leq c_{0}^{2} /(2 d)$. If $\left|u^{0}\right|_{H} \leq R$, then for all $n \in \mathbb{N}$ such that $n \tau \geq 2 t_{0}(R)$, we have

$$
\left|u^{n}\right|_{H} \leq \rho_{0}^{\prime} .
$$


Proof. We multiply (4.1) by $u^{n+1}$ and integrate over $\Omega$. We obtain

$$
\frac{1}{\tau}\left(u^{n+1}-u^{n}, u^{n+1}\right)_{H}+d\left\|u^{n+1}\right\|_{V}^{2}+\int_{\Omega} g\left(u^{n+1}\right) u^{n+1} d x=0 .
$$

We use (4.5) and inequality (3.10) with $q=2$. We find

$$
\frac{1}{2 \tau}\left(\left|u^{n+1}\right|_{H}^{2}-\left|u^{n}\right|_{H}^{2}\right)+d\left\|u^{n+1}\right\|_{V}^{2} \leq c_{2}^{\prime}|\Omega|, \quad n \geq 0 .
$$

We infer from the Poincaré inequality (3.3) that

$$
\left(1+\frac{2 d \tau}{c_{0}^{2}}\right)\left|u^{n+1}\right|_{H}^{2} \leq\left|u^{n}\right|_{H}^{2}+c_{2}^{\prime \prime} \tau, \quad n \geq 0
$$

Let $a=1+\left(2 d \tau / c_{0}^{2}\right)$. By induction, we obtain that

$$
\left|u^{n}\right|_{H}^{2} \leq a^{-n}\left|u^{0}\right|_{H}^{2}+c_{2}^{\prime \prime} \tau \frac{1-a^{-n}}{a-1}, \quad n \geq 0 .
$$

We note that $\exp (s / 2) \leq 1+s$, for all $s \in[0,1]$. Applying this to $s=2 d \tau / c_{0}^{2} \leq 1$, we see that $a^{-1} \leq \exp \left(-d \tau / c_{0}^{2}\right)$, and we find

$$
\left|u^{n}\right|_{H}^{2} \leq \exp \left(-n d \tau / c_{0}^{2}\right)\left|u^{0}\right|_{H}^{2}+\frac{c_{2}^{\prime \prime} c_{0}^{2}}{2 d}\left(1-\exp \left(-n d \tau / c_{0}^{2}\right)\right), \quad n \geq 0 .
$$

This implies (4.6).

We will use the following lemma from [28].

Lemma 4.3 (Discrete Uniform Gronwall Lemma). Let $n_{0}, N \in \mathbb{N}, a_{1}, a_{2}, a_{3}, \tau, r^{\prime}>0$ and $\left(d^{n}\right),\left(g^{n}\right),\left(h^{n}\right)$ be three sequences of nonnegative real numbers which satisfy

$$
\frac{d^{n+1}-d^{n}}{\tau} \leq g^{n} d^{n}+h^{n}, \quad \forall n \geq n_{0}
$$

and

$$
\tau \sum_{n=k_{0}}^{k_{0}+N} g^{n} \leq a_{1}, \quad \tau \sum_{n=k_{0}}^{k_{0}+N} h^{n} \leq a_{2}, \quad \tau \sum_{n=k_{0}}^{k_{0}+N} d^{n} \leq a_{3},
$$

for all $k_{0} \geq n_{0}$, with $r^{\prime}=\tau N>0$. Then

$$
d^{n} \leq\left(a_{2}+\frac{a_{3}}{r^{\prime}}\right) \exp \left(a_{1}\right), \quad \forall n \geq n_{0}+N .
$$

The following lemma will prove useful.

Lemma 4.4. Assume that $\tau \leq 1 /\left(4 c_{1}^{\prime}\right)$. Then

$$
\left\|u^{n+1}\right\|_{V}^{2}+\left\|u^{n+1}-u^{n}\right\|_{V}^{2} \leq\left(1+4 c_{1}^{\prime} \tau\right)\left\|u^{n}\right\|_{V}^{2}, \quad n \geq 0 .
$$

Note that if $u^{0} \notin V$, then (4.8) is valid for $n \geq 1$ only.

Proof. We multiply (4.1) by $-\Delta u^{n+1}$ and integrate over $\Omega$. This yields

$$
\frac{1}{\tau}\left(\nabla\left(u^{n+1}-u^{n}\right), \nabla u^{n+1}\right)_{H}+d\left|\Delta u^{n+1}\right|_{H}^{2}+\int_{\Omega} g^{\prime}\left(u^{n+1}\right)\left|\nabla u^{n+1}\right|^{2} d x=0 .
$$

Using (4.5) and (3.6), we obtain

$$
\frac{1}{\tau}\left(\left\|u^{n+1}\right\|_{V}^{2}-\left\|u^{n}\right\|_{V}^{2}+\left\|u^{n+1}-u^{n}\right\|_{V}^{2}\right) \leq 2 c_{1}^{\prime}\left\|u^{n+1}\right\|_{V}^{2}, \quad n \geq 0 .
$$


We note that

$$
1 \leq \frac{1}{1-s} \leq 1+2 s, \quad \forall s \in[0,1 / 2],
$$

and we apply this with $s=2 c_{1}^{\prime} \tau$. We find (4.8). These formal computations are fully justified for smooth solutions, and (4.8) is valid in the general case by regularization (proceed as in Lemma 4.6 and use that $S_{\tau}$ is continuous from $V$ into $V$ ).

Proposition 4.5 (Absorbing set in $V$ ). Assume that $\tau \leq \tau_{1}$ where

$$
\tau_{1}=\min \left\{c_{0}^{2} /(2 d), r / 2,1 /\left(4 c_{1}^{\prime}\right)\right\}>0 .
$$

If $\left|u^{0}\right|_{H} \leq R$, then for all $n \in \mathbb{N}$ such that $n \tau \geq 2 t_{0}(R)+2 r$, we have

$$
\left\|u^{n}\right\|_{V} \leq \hat{\rho}_{1}
$$

where

$$
\hat{\rho}_{1}^{2}=\frac{\hat{\kappa}_{1}}{r} \exp \left(8 c_{1}^{\prime} r\right), \quad \hat{\kappa}_{1}=\frac{2 c_{2}^{\prime \prime} r+\rho_{0}^{\prime 2}}{d} .
$$

Proof. Let $k_{0}, N \in \mathbb{N} \backslash\{0\}$. Summing (4.7) from $n=k_{0}-1$ to $k_{0}+N-1$, we obtain

$$
\left|u^{k_{0}+N}\right|_{H}^{2}+2 \tau d \sum_{n=k_{0}}^{k_{0}+N}\left\|u^{n}\right\|_{V}^{2} \leq c_{2}^{\prime \prime} \tau(N+1)+\left|u^{k_{0}-1}\right|_{H}^{2} .
$$

If $k_{0} \tau \geq 2 t_{0}(R)+\tau$, from (4.6) we infer that

$$
2 \tau d \sum_{n=k_{0}}^{k_{0}+N}\left\|u^{n}\right\|_{V}^{2} \leq c_{2}^{\prime \prime} \tau(N+1)+\rho_{0}^{\prime 2} .
$$

Let $n_{0} \tau \geq 2 t_{0}(R)+\tau$ and $N=\left[r / \tau \mid \geq 2\right.$. We set $r^{\prime}=N \tau \in[r-\tau, r]$ and

$$
\hat{\kappa}_{1}^{\prime}=\frac{c_{2}^{\prime \prime}\left(r^{\prime}+\tau\right)+\rho_{0}^{\prime 2}}{2 d} .
$$

Using (4.11), (4.8) and Lemma 4.3, we obtain

$$
\left\|u^{n}\right\|_{V}^{2} \leq \frac{\hat{\kappa}_{1}^{\prime}}{r^{\prime}} \exp \left(4 c_{1}^{\prime}\left(r^{\prime}+\tau\right)\right), \quad \forall n \geq n_{0}+N .
$$

This implies (4.10).

Lemma 4.6. Let $q \geq 2$ and assume that $u^{n} \in L^{q}(\Omega)$ for some $n \geq 0$. Then $u^{n+1}$ belongs to $L^{2 p+q-2}(\Omega)$ and satisfies

$$
\frac{1}{\tau}\left(\left\|u^{n+1}\right\|_{L^{q}}^{q}-\left\|u^{n}\right\|_{L^{q}}^{q}\right)+\frac{q}{2} b_{2 p-1}\left\|u^{n+1}\right\|_{L^{2 p+q-2}}^{2 p+q-2} \leq c_{q}^{\prime \prime} .
$$

Proof. For simplicity, we denote $u=u^{n}$ and $v=u^{n+1}=S_{\tau} u$. First assume that $u \in C_{c}^{\infty}(\Omega)$. Then, by elliptic regularity, $v \in W^{2, s}(\Omega) \cap W_{0}^{1, s}(\Omega)$ for all $s>0$, and in particular, $v \in L^{\infty}(\Omega)$. We multiply (4.2) by $|v|^{q-2} v$ and integrate over $\Omega$. This yields

$$
\frac{1}{\tau}\left(v-u,|v|^{q-2} v\right)_{H}+(q-1) d \int_{\Omega}|\nabla v|^{2}|v|^{q-2} d x+\int_{\Omega} g(v)|v|^{q-2} v d x=0 .
$$

Let $f(s)=|s|^{q} / q$. Then $f \in C^{2}(\mathbb{R})$ with $f^{\prime \prime}(s) \geq 0$ on $\mathbb{R}$, so by the Taylor-Lagrange formula, for all $s_{1}, s_{2} \in \mathbb{R}$, we have

$$
f\left(s_{1}\right)-f\left(s_{2}\right)=\left(s_{1}-s_{2}\right) f^{\prime}\left(s_{2}\right)+\frac{\left(s_{1}-s_{2}\right)^{2}}{2} f^{\prime \prime}\left(\xi_{s_{1}, s_{2}}\right) \geq\left(s_{1}-s_{2}\right) f^{\prime}\left(s_{2}\right) .
$$


We apply this with $s_{1}=u(x), s_{2}=v(x)$ and we integrate over $\Omega$. We obtain

$$
\frac{1}{q} \int_{\Omega}|u|^{q} d x-\frac{1}{q} \int_{\Omega}|v|^{q} d x \geq \int_{\Omega}(u-v)|v|^{q-2} v d x .
$$

We infer from (4.13) and (3.10) that (4.12) is true when $u \in C_{c}^{\infty}(\Omega)$. If $u \in L^{q}(\Omega)$, we obtain (4.12) by considering a sequence $u_{\varepsilon} \in C_{c}^{\infty}(\Omega)$ such that $u_{\varepsilon} \rightarrow u$ in $L^{q}(\Omega)$.

As a consequence, we have:

Lemma 4.7. Let $q \geq 2$. If $u^{0} \in L^{q}(\Omega)$, then

$$
\left\|u^{n}\right\|_{L^{q}}^{q} \leq\left\|u^{0}\right\|_{L^{q}}^{q}+n \tau c_{q}^{\prime \prime}, \quad \forall n \geq 0 .
$$

We introduce the sequence $\left(\hat{\rho}_{i}^{\prime}\right)$ defined by $\hat{\rho}_{0}^{\prime}=\rho_{0}^{\prime}$ and, for $i \geq 0$,

$$
\hat{\rho}_{i+1}^{\prime a_{i+1}}=\frac{\hat{\kappa}_{i}^{\prime}}{r}+2 c_{a_{i+1}}^{\prime \prime} r \quad \text { with } \quad \hat{\kappa}_{i}^{\prime}=\frac{4}{a_{i} b_{2 p-1}}\left(2 c_{a_{i}}^{\prime \prime} r+\hat{\rho}_{i}^{\prime a_{i}}\right) .
$$

Proposition 4.8 (Absorbing set in $L^{q}$ ). Assume that $\tau \leq \min \left\{1 / c_{1}^{\prime}, c_{0}^{2} /(2 d), r / 2\right\}$. If $\left|u^{0}\right|_{H} \leq R$ and $i \in \mathbb{N}$, then for all $n \in \mathbb{N}$ such that $n \tau \geq \hat{t}_{i}(R)$, we have

$$
\left\|u^{n}\right\|_{L^{a_{i}}} \leq \hat{\rho}_{i}^{\prime}
$$

where $\hat{t}_{i}(R)=2 t_{0}(R)+3$ ir does not depend on $\tau$.

Proof. We proceed by induction. We first note that (4.14) is satisfied for $i=0$ by Proposition 4.2. Let $i \geq 0$ and assume that (4.14) holds for $n \tau \geq \hat{t}_{i}(R)$. Let $k_{0} \tau \geq \hat{t}_{i}(R)+\tau$ and $N=[r / \tau] \geq 2$. We apply Lemma 4.6 with $q=a_{i}$ and we add the resulting inequality from $n=k_{0}-1$ to $n=k_{0}+N-1$. This yields

$$
\frac{a_{i}}{2} b_{2 p-1} \tau \sum_{n=k_{0}}^{k_{0}+N}\left\|u^{n}\right\|_{L^{a_{i+1}}}^{a_{i+1}} \leq c_{a_{i}}^{\prime \prime} \tau(N+1)+\left\|u^{k_{0}-1}\right\|_{L^{a_{i}}}^{a_{i}}
$$

We set $r^{\prime}=N \tau \in[r-\tau, r]$. Using the induction assumption, we see that

$$
\tau \sum_{n=k_{0}}^{k_{0}+N}\left\|u^{n}\right\|_{L^{a_{i}}}^{a_{i}} \leq \hat{\kappa}_{i}^{\prime \prime}, \quad \hat{\kappa}_{i}^{\prime \prime}=\frac{2}{a_{i} b_{2 p-1}}\left(c_{a_{i}}^{\prime \prime}\left(r^{\prime}+\tau\right)+\hat{\rho}_{i}^{\prime a_{i}}\right) .
$$

For $n \geq k_{0}+1$, we may apply Lemma 4.6 with $q=a_{i+1}$ and we obtain

$$
\frac{1}{\tau}\left(\left\|u^{n+1}\right\|_{L^{a_{i+1}}}^{a_{i+1}}-\left\|u^{n}\right\|_{L^{a_{i+1}}}^{a_{i+1}}\right) \leq c_{a_{i+1}}^{\prime \prime} .
$$

Let $n_{0} \tau \geq \hat{t}_{i}(R)+\tau$. We infer from (4.15), (4.16) and Lemma 4.3 that

$$
\left\|u^{n}\right\|_{L^{a_{i+1}}}^{a_{a_{1}}} \leq \frac{\hat{\kappa}_{i}^{\prime \prime}}{r^{\prime}}+c_{a_{i+1}}^{\prime \prime}\left(r^{\prime}+\tau\right), \quad \forall n \geq n_{0}+N+1 .
$$

This implies (4.14).

Lemma 4.9. If $u^{0} \in V \cap L^{2 p}(\Omega)$, then

$$
\left\|u^{n}\right\|_{V}^{2}+\frac{b_{2 p-1}}{2 d p}\left\|u^{n}\right\|_{L^{2 p}}^{2 p}+\frac{1}{d \tau} \sum_{k=0}^{n-1}\left|u^{k+1}-u^{k}\right|_{H}^{2} \leq\left\|u^{0}\right\|_{V}^{2}+Q_{1}\left(\left\|u^{0}\right\|_{L^{2 p}}\right), \quad \forall n \geq 0,
$$

where $Q_{1}$ is the monotonic function independent of $\tau$ defined by (3.23). 
Proof. We multiply (4.1) by $u^{n+1}-u^{n}$ and integrate over $\Omega$. We obtain

$$
\frac{\left|u^{n+1}-u^{n}\right|_{H}^{2}}{\tau}+d\left(\nabla u^{n+1}, \nabla\left(u^{n+1}-u^{n}\right)\right)_{H}+\int_{\Omega} g\left(u^{n+1}\right)\left(u^{n+1}-u^{n}\right) d x=0,
$$

for all $n \geq 0$. By the Taylor-Lagrange formula, for all $s_{1}, s_{2} \in \mathbb{R}$

$$
G\left(s_{1}\right)-G\left(s_{2}\right)=\left(s_{1}-s_{2}\right) g\left(s_{2}\right)+\frac{\left(s_{1}-s_{2}\right)^{2}}{2} g^{\prime}\left(\xi_{s_{1}, s_{2}}\right),
$$

for some $\xi_{s_{1}, s_{2}} \in \mathbb{R}$, and so, using (3.6), we have

$$
G\left(s_{1}\right)-G\left(s_{2}\right) \geq\left(s_{1}-s_{2}\right) g\left(s_{2}\right)-c_{1}^{\prime} \frac{\left(s_{1}-s_{2}\right)^{2}}{2} .
$$

We choose $s_{1}=u^{n}(x), s_{2}=u^{n+1}(x)$, and we integrate over $\Omega$. We infer from the resulting inequality, from (4.18) and (4.5) that

$$
\left(\frac{1}{\tau}-\frac{c_{1}^{\prime}}{2}\right)\left|u^{n+1}-u^{n}\right|_{H}^{2}+\frac{d}{2}\left(\left\|u^{n+1}\right\|_{V}^{2}-\left\|u^{n}\right\|_{V}^{2}\right)+\int_{\Omega} G\left(u^{n+1}\right) d x-\int_{\Omega} G\left(u^{n}\right) d x \leq 0,
$$

for all $n \geq 0$. By summation, we obtain

$$
\frac{d}{2}\left\|u^{n}\right\|_{V}^{2}+\int_{\Omega} G\left(u^{n}\right) d x+\frac{1}{2 \tau} \sum_{k=0}^{n-1}\left|u^{k+1}-u^{k}\right|_{H}^{2} \leq \frac{d}{2}\left\|u^{0}\right\|_{V}^{2}+\int_{\Omega} G\left(u^{0}\right) d x,
$$

for all $n \geq 0$, where we used that $1 / \tau-c_{1}^{\prime} / 2 \geq 1 /(2 \tau)$. Using (3.9), we conclude that (4.17) holds with $Q_{1}$ defined by (3.23).

4.3. Estimates for the difference of solutions, uniform with $\tau$. Let $\left(u^{n}\right)$ and $\left(\hat{u}^{n}\right)$ be two sequences generated by the scheme (4.1) and corresponding to the initial condition $u^{0}$ and $\hat{u}^{0}$ respectively. We denote $v^{n}=u^{n}-\hat{u}^{n}$ their difference, which satisfies

$$
\frac{v^{n+1}-v^{n}}{\tau}-d \Delta v^{n+1}+g\left(u^{n+1}\right)-g\left(\hat{u}^{n+1}\right)=0, \quad \forall n \geq 0 .
$$

Lemma 4.10. Assume that $\tau \leq 1 /\left(4 c_{1}^{\prime}\right)$. Then

$$
\left|v^{n}\right|_{H}^{2}+2 d \tau \sum_{k=0}^{n-1}\left\|v^{k+1}\right\|_{V}^{2} \leq \exp \left(4 c_{1}^{\prime} n \tau\right)\left|v^{0}\right|_{H}^{2}, \quad \forall n \geq 0 .
$$

Proof. We multiply (4.19) by $v^{n+1}$, integrate over $\Omega$, and we use (4.5) and (3.7). We find

$$
\frac{1}{2 \tau}\left(\left|v^{n+1}\right|_{H}^{2}-\left|v^{n}\right|_{H}^{2}\right)+d\left\|v^{n+1}\right\|_{V}^{2} \leq c_{1}^{\prime}\left|v^{n+1}\right|_{H}^{2}, \quad \forall n \geq 0 .
$$

From (4.9), we infer that

$$
\left|v^{n+1}\right|_{H}^{2}+2 d \tau\left\|v^{n+1}\right\|_{V}^{2} \leq\left(1+4 c_{1}^{\prime} \tau\right)\left|v^{n}\right|_{H}^{2}, \quad \forall n \geq 0 .
$$

We apply

$$
1+s \leq \exp (s), \quad \forall s \in \mathbb{R},
$$

to $s=4 c_{1}^{\prime} \tau$ and we obtain (4.20) by induction.

Proposition 4.11 (Bound on bounded sets). Assume that $\tau \leq 1 /\left(4 c_{1}^{\prime}\right)$. For all $T>0$ and for all $R>0$, there exists a constant $C(T, R)$ independent of $\tau$ such that $\left|u^{0}\right|_{H} \leq R$ and $0 \leq n \tau \leq T$ imply $\left|u^{n}\right|_{H} \leq C(T, R)$. 
Proof. Let $T>0, R>0,\left|u^{0}\right|_{H} \leq R$ and $0 \leq n \tau \leq T$. We choose $\hat{u}^{0}=0$. Using successively the triangle inequality, (4.20) and the Cauchy-Schwarz inequality, we find

$$
\begin{aligned}
\left|u^{n}\right|_{H} & \leq\left|u^{n}-\hat{u}^{n}\right|_{H}+\left|\hat{u}^{n}-\hat{u}^{0}\right|_{H} \\
& \leq \exp \left(2 c_{1}^{\prime} T\right) R+\sum_{k=0}^{n-1}\left|\hat{u}^{k+1}-\hat{u}^{k}\right|_{H} \\
& \leq \exp \left(2 c_{1}^{\prime} T\right) R+(n \tau)^{1 / 2}\left(\frac{1}{\tau} \sum_{k=0}^{n-1}\left|\hat{u}^{k+1}-\hat{u}^{k}\right|_{H}^{2}\right)^{1 / 2} .
\end{aligned}
$$

We conclude with (4.17) that

$$
\left|u^{n}\right|_{H} \leq \exp \left(2 c_{1}^{\prime} T\right) R+\left(T d \tilde{Q}_{1}(0)\right)^{1 / 2}
$$

and this proves the assertion.

Proposition 4.12 (Smoothing property). Assume that Case 1 holds and that

$$
\tau \leq \min \left\{1 / c_{1}^{\prime}, 1 /\left(2 \tilde{h}_{1}\left(R_{1}\right)\right)\right\}
$$

where $\tilde{h}_{1}$ (independent of $\tau$ ) is defined by (3.31). If $\left\|u^{0}\right\|_{V} \leq R_{1}$ and $\left\|\hat{u}^{0}\right\|_{V} \leq R_{1}$, then for all $n \geq 1$, we have

$$
n \tau\left\|v^{n}\right\|_{V}^{2} \leq \frac{1}{2 d} \exp \left(4 c_{1}^{\prime} n \tau\right) \exp \left(2 \tilde{h}_{1}\left(R_{1}\right)(n+1) \tau\right)\left|v^{0}\right|_{H}^{2},
$$

Proof. Lemma 4.9 shows that

$$
\left\|u^{n}\right\|_{V} \leq \tilde{Q}_{1}\left(R_{1}\right) \quad \text { and } \quad\left\|\hat{u}^{n}\right\|_{V} \leq \tilde{Q}_{1}\left(R_{1}\right) \quad \forall n \geq 0,
$$

where $\tilde{Q}_{1}$, defined by (3.30), is independent of $\tau$. We multiply (4.19) by $\left(v^{n+1}-v^{n}\right)$ and we integrate over $\Omega$. We find

$\frac{\left|v^{n+1}-v^{n}\right|_{H}^{2}}{\tau}+d\left(\nabla v^{n+1}, \nabla\left(v^{n+1}-v^{n}\right)\right)_{H}=-\int_{\Omega}\left[g\left(u^{n+1}\right)-g\left(\hat{u}^{n+1}\right)\right]\left(v^{n+1}-v^{n}\right) d x$,

for all $n \geq 0$. We multiply this by $n$, we use (4.5) and (3.11), and we obtain

$$
n \frac{\left|v^{n+1}-v^{n}\right|_{H}^{2}}{\tau}+\frac{n d}{2}\left(\left\|v^{n+1}\right\|_{V}^{2}-\left\|v^{n}\right\|_{V}^{2}\right) \leq n h_{1}\left(\tilde{Q}_{1}\left(R_{1}\right)\right)\left\|v^{n+1}\right\|_{V}\left|v^{n+1}-v^{n}\right|_{H},
$$

for all $n \geq 0$. Next, we use Young's inequality and the identity

$$
n\left(\left\|v^{n+1}\right\|_{V}^{2}-\left\|v^{n}\right\|_{V}^{2}\right)=\left((n+1)\left\|v^{n+1}\right\|_{V}^{2}-n\left\|v^{n}\right\|_{V}^{2}\right)-\left\|v^{n+1}\right\|_{V}^{2},
$$

and we find

$$
(n+1)\left\|v^{n+1}\right\|_{V}^{2}-n\left\|v^{n}\right\|_{V}^{2} \leq \tilde{h}_{1}\left(R_{1}\right) \tau(n+1)\left\|v^{n+1}\right\|_{V}^{2}+\left\|v^{n+1}\right\|_{V}^{2},
$$

for all $n \geq 0$. Let $\alpha_{n}=n\left\|v^{n}\right\|_{V}^{2}$. We infer from (4.9) that

$$
\alpha_{n+1} \leq\left(1+2 \tilde{h}_{1}\left(R_{1}\right) \tau\right)\left(\alpha_{n}+\left\|v^{n+1}\right\|_{V}^{2}\right), \quad \forall n \geq 0 .
$$

Using $\alpha_{0}=0$, we find by induction that

$$
\alpha_{n} \leq\left(1+2 \tilde{h}_{1}\left(R_{1}\right) \tau\right)^{n+1}\left(\sum_{k=0}^{n-1}\left\|v^{k+1}\right\|_{V}^{2}\right), \quad \forall n \geq 1 .
$$

We infer (4.22) from (4.20) and from (4.21) applied to $s=2 \tilde{h}_{1}\left(R_{1}\right) \tau$. 
Proposition 4.13 (Smoothing property). Let $T>0$. Assume that Case 2 holds and that $\tau \leq \min \left\{1 / c_{1}^{\prime}, 1 /\left(2 \tilde{h}_{2}\left(T, R_{2}\right)\right)\right\}$ where $\tilde{h}_{2}$ (independent of $\tau$ ) is defined by (3.35). If $\left\|u^{0}\right\|_{L^{I(2 p-2)}} \leq R_{2}$ and $\left\|\hat{u}^{0}\right\|_{L^{I(2 p-2)}} \leq R_{2}$, then for all $1 \leq n \leq[T / \tau]$ we have

$$
n \tau\left\|v^{n}\right\|_{V}^{2} \leq \frac{1}{2 d} \exp \left(4 c_{1}^{\prime} n \tau\right) \exp \left(2 \tilde{h}_{2}\left(T, R_{2}\right)(n+1) \tau\right)\left|v^{0}\right|_{H}^{2} .
$$

Proof. Let $T>0$ and set $q=I(2 p-2)$. Lemma 4.7 shows that

$$
\left\|u^{n}\right\|_{L^{q}} \leq \tilde{Q}_{2}\left(T, R_{2}\right) \quad \text { and } \quad\left\|\hat{u}^{n}\right\|_{L^{q}} \leq \tilde{Q}_{2}\left(T, R_{2}\right), \quad \forall 0 \leq n \leq[T / \tau],
$$

where $\tilde{Q}_{2}$ (independent of $\tau$ ) is defined by (3.34). Arguing as in the proof of Proposition 4.12 and using (3.12), we find

$$
(n+1)\left\|v^{n+1}\right\|_{V}^{2}-n\left\|v^{n}\right\|_{V}^{2} \leq \tilde{h}_{2}\left(T, R_{2}\right) \tau(n+1)\left\|v^{n+1}\right\|_{V}^{2}+\left\|v^{n+1}\right\|_{V}^{2},
$$

for all $0 \leq n<[T / \tau]$. We conclude similarly.

\section{Finite time Uniform ERror estimate}

For the error estimate on a finite time interval, we follow the methodology in [31].

We assume that $\tau \in\left(0,1 /\left(4 c_{1}^{\prime}\right)\right]$ and we consider a sequence $\left(u^{n}\right)$ generated by (4.1). We first derive a very useful estimate. Using (4.21) with $s=4 c_{1}^{\prime} \tau$, from (4.8) we infer by induction that

$$
\left\|u^{n}\right\|_{V}^{2}+\sum_{k=0}^{n-1}\left\|u^{k+1}-u^{k}\right\|_{V}^{2} \leq \exp \left(4 c_{1}^{\prime} n \tau\right)\left\|u^{0}\right\|_{V}^{2}, \quad \forall n \geq 0 .
$$

To the sequence $\left(u^{n}\right)$, we associate two functions $u_{\tau}, \bar{u}_{\tau}: \mathbb{R}_{+} \rightarrow H$, namely

$$
u_{\tau}(t)=u^{n}+\frac{t-n \tau}{\tau}\left(u^{n+1}-u^{n}\right), \quad t \in[n \tau,(n+1) \tau),
$$

and

$$
\bar{u}_{\tau}(t)=u^{n+1}, \quad t \in[n \tau,(n+1) \tau) .
$$

We assume that $u^{0} \in V \cap L^{2 p}(\Omega)$. Then, by definition, every $u^{n}$ belongs to $V \cap L^{2 p}(\Omega)$, so that $u_{\tau} \in C^{0}\left(\mathbb{R}_{+} ; V \cap L^{2 p}(\Omega)\right), \partial_{t} u_{\tau} \in L_{l o c}^{\infty}\left(\mathbb{R}_{+} ; V \cap L^{2 p}(\Omega)\right)$ and $\bar{u}_{\tau} \in L_{l o c}^{\infty}\left(\mathbb{R}_{+} ; V \cap\right.$ $L^{2 p}(\Omega)$ ). The scheme (4.1) can be rewritten

$$
\partial_{t} u_{\tau}-d \Delta \bar{u}_{\tau}+g\left(\bar{u}_{\tau}\right)=0, \quad \text { a.e. } t \geq 0
$$

or equivalently,

$$
\partial_{t} u_{\tau}-d \Delta u_{\tau}+g\left(u_{\tau}\right)=-d \Delta\left(u_{\tau}-\bar{u}_{\tau}\right)+\left[g\left(u_{\tau}\right)-g\left(\bar{u}_{\tau}\right)\right], \quad \text { a.e. } t \geq 0 .
$$

Let $u$ denote a solution of (3.1)-(3.2) with $u_{0} \in V \cap L^{2 p}(\Omega)$, and set $e_{\tau}=u_{\tau}-u$. Subtracting (3.1) from (5.2), we find that

$$
\partial_{t} e_{\tau}-d \Delta e_{\tau}+g\left(u_{\tau}\right)-g(u)=-d \Delta\left(u_{\tau}-\bar{u}_{\tau}\right)+\left[g\left(u_{\tau}\right)-g\left(\bar{u}_{\tau}\right)\right], \quad \text { a.e. } t \geq 0 .
$$

We multiply by $e_{\tau}$, integrate over $\Omega$ and use (3.7). We obtain

$$
\begin{aligned}
\frac{1}{2} \frac{d}{d t}\left|e_{\tau}\right|_{H}^{2}+d\left\|e_{\tau}\right\|_{V}^{2} \leq & c_{1}^{\prime}\left|e_{\tau}\right|_{H}^{2}+d\left\|u_{\tau}-\bar{u}_{\tau}\right\|_{V}\left\|e_{\tau}\right\|_{V} \\
& +\int_{\Omega}\left|g\left(u_{\tau}\right)-g\left(\bar{u}_{\tau}\right) \| e_{\tau}\right| d x .
\end{aligned}
$$


Theorem 5.1. Assume that Case 1 holds. For all $T>0$ and $R_{1}>0$, there is a constant $C\left(T, R_{1}\right)$ independent of $\tau$ such that $u^{0}=u_{0}$ and $\left\|u^{0}\right\|_{V} \leq R_{1}$ imply

$$
\sup _{t \in[0, N \tau]}\left|e_{\tau}(t)\right|_{H} \leq C\left(T, R_{1}\right) \tau^{1 / 2}, \text { where } N=[T / \tau] .
$$

Proof. From Lemma 4.9, we infer that $\left\|u^{n}\right\|_{V} \leq \tilde{Q}\left(R_{1}\right)$ for all $n \geq 0$, where $\tilde{Q}_{1}$ (independent of $\tau$ ) is defined by (3.30). Thus,

$$
\left\|u_{\tau}(t)\right\|_{V} \leq \tilde{Q}_{1}\left(R_{1}\right) \quad \text { and } \quad\left\|\bar{u}_{\tau}(t)\right\|_{V} \leq \tilde{Q}_{1}\left(R_{1}\right), \quad \forall t \geq 0 .
$$

Using (5.3), (3.11) and Young's inequality, we infer that

$$
\frac{d}{d t}\left|e_{\tau}(t)\right|_{H}^{2} \leq C_{3}\left(R_{1}\right)\left|e_{\tau}(t)\right|_{H}^{2}+d\left\|u_{\tau}(t)-\bar{u}_{\tau}(t)\right\|_{V}^{2}, \quad \text { a.e. } t \geq 0
$$

for some constant $C_{3}\left(R_{1}\right)$ independent of $\tau$. Let $T>0$ and set $N=[T / \tau]$. Using $e_{\tau}(0)=0$, the classical Gronwall lemma yields

$$
\left|e_{\tau}(t)\right|_{H}^{2} \leq d \exp \left(C_{3}\left(R_{1}\right) T\right) \int_{0}^{N \tau}\left\|u_{\tau}(s)-\bar{u}_{\tau}(s)\right\|_{V}^{2} d s, \quad \forall t \in[0, N \tau] .
$$

On $[n \tau,(n+1) \tau)$, we have $\left\|u_{\tau}(s)-\bar{u}_{\tau}(s)\right\|_{V} \leq\left\|u^{n+1}-u^{n}\right\|_{V}$, so that

$$
\int_{0}^{N \tau}\left\|u_{\tau}(s)-\bar{u}_{\tau}(s)\right\|_{V}^{2} d s \leq \tau \sum_{n=0}^{N-1}\left\|u^{n+1}-u^{n}\right\|_{V}^{2} .
$$

This shows, using (5.1) and (5.4), that

$$
\left|e_{\tau}(t)\right|_{H}^{2} \leq\left[d \exp \left(C_{3}\left(R_{1}\right) T\right) \exp \left(4 c_{1}^{\prime} T\right) R_{1}^{2}\right] \tau, \quad \forall t \in[0, N \tau] .
$$

The proof is complete.

Theorem 5.2. Assume that Case 2 holds. For all $T>0$ and $R_{1}, R_{2}>0$ there is a constant $C\left(T, R_{1}, R_{2}\right)$ independent of $\tau$ such that $u^{0}=u_{0},\left\|u^{0}\right\|_{V} \leq R_{1}$ and $\left\|u^{0}\right\|_{L^{I(2 p-2)}}^{I(2 p-2)} \leq R_{2}$ imply

$$
\sup _{t \in[0, N \tau]}\left|e_{\tau}(t)\right|_{H} \leq C\left(T, R_{1}, R_{2}\right) \tau^{1 / 2}
$$

Proof. Let $T>0$, set $N=[T / \tau]$ and $q=I(2 p-2)$. Lemma 4.7 shows that $\left\|u^{n}\right\|_{L^{q}} \leq \tilde{Q}_{2}\left(T+1 / c_{1}^{\prime}, R_{2}\right)$ for all $0 \leq n \leq N+1$, where $\tilde{Q}_{2}$ (independent of $\tau$ ) is defined by (3.34). Thus,

$\left\|u_{\tau}(t)\right\|_{L^{q}} \leq \tilde{Q}_{2}\left(T+1 / c_{1}^{\prime}, R_{2}\right) \quad$ and $\quad\left\|\bar{u}_{\tau}(t)\right\|_{L^{q}} \leq \tilde{Q}_{2}\left(T+1 / c_{1}^{\prime}, R_{2}\right), \quad \forall t \in[0, N \tau]$.

Using (5.3), (3.12) and Young's inequality, we obtain

$$
\frac{d}{d t}\left|e_{\tau}(t)\right|_{H}^{2} \leq C_{4}\left(T, R_{2}\right)\left|e_{\tau}(t)\right|_{H}^{2}+d\left\|u_{\tau}(t)-\bar{u}_{\tau}(t)\right\|_{V}^{2}, \quad \forall t \in[0, N \tau] .
$$

Using $e_{\tau}(0)=0$, the classical Gronwall lemma yields

$$
\left|e_{\tau}(t)\right|_{H}^{2} \leq d \exp \left(C_{4}\left(T, R_{2}\right) T\right) \int_{0}^{N \tau}\left\|u_{\tau}(s)-\bar{u}_{\tau}(s)\right\|_{V}^{2} d s, \quad \forall t \in[0, N \tau] .
$$

From (5.1) we infer that

$$
\left|e_{\tau}(t)\right|_{H}^{2} \leq\left[d \exp \left(C_{4}\left(T, R_{2}\right) T\right) \exp \left(4 c_{1}^{\prime} T\right) R_{1}^{2}\right] \tau, \quad \forall t \in[0, N \tau]
$$




\section{The CONVERGENCE RESUlt}

Theorem 6.1. Let $H=L^{2}(\Omega)$ and $\tau_{0}=\min \left\{c_{0}^{2} /(2 d), 1 /\left(4 c_{1}^{\prime}\right)\right\}$. The continuous semi-group $\left\{S_{0}(t), t \in \mathbb{R}_{+}\right\}$associated to (3.1) and the family of discrete semi-groups $\left\{S_{\tau}^{n}, n \in \mathbb{N}\right\}, \tau \in\left(0, \tau_{0}\right]$, associated to (4.1) satisfy the conclusions of Theorem 2.5 with $\tau_{0}^{\prime}=\tau_{0}$.

Proof. We apply Theorem 2.5 with $V=H_{0}^{1}(\Omega)$ which is compactly imbedded in $H$ [18]. If Case 1 holds, we choose $r \geq 1 /\left(2 c_{1}^{\prime}\right)$ and we consider the set

$$
\mathcal{B}=\left\{w \in H:\|w\|_{V} \leq \hat{\rho}_{1}\right\}
$$

which is absorbing in $H$, uniformly with respect to $\tau \in\left[0, \tau_{0}\right]$ since $\rho_{1}<\hat{\rho}_{1}$. The estimates of Sections 3-5 show that assumptions (H1)-(H9) are satisfied with $\beta=\gamma=$ $1 / 2$ and, in (H6), $\bar{\tau}(T)=\bar{\tau}=\min \left\{\tau_{0}, 1 /\left(2 \tilde{h}_{1}\left(R_{1}\right)\right)\right\}$, (cf. (3.31)) and the conclusions of Theorem 2.5 follow for some $\tau_{0}^{\prime} \in\left(0, \tau_{0}\right]$ small enough.

For $\tau \in\left[\tau_{0}^{\prime}, \tau_{0}\right]$, we set $T_{0}=t(\mathcal{B})+\tau_{0}(\mathrm{cf}$. $(\mathrm{H} 5))$ and $\tilde{S}_{\tau}=S_{\tau}^{\left[T_{0} / \tau\right]}$, so that $\tilde{S}_{\tau} \mathcal{B} \subset \mathcal{B}$. By (4.3), $\tilde{S}_{\tau}$ satisfies a smoothing property on $\mathcal{B}$ with a Lipschitz constant bounded by a constant $\Lambda=\Lambda\left(T_{0}, \tau_{0}, \tau_{0}^{\prime}, c_{0}, d\right)$ independent of $\tau$. Proposition 1 in [10] shows that the map $\tilde{S}_{\tau}: \mathcal{B} \rightarrow \mathcal{B}$ possesses an exponential attractor $\mathcal{M}_{\tau}^{d}$, i.e. a compact and positively invariant subset of $\mathcal{B}$ which has finite fractal dimension and which satisfies

$$
\operatorname{dist}_{H}\left(\tilde{S}_{\tau}^{n} \mathcal{B}, \mathcal{M}_{\tau}^{d}\right) \leq 2\|\mathcal{B}\|_{H} 2^{-n}, \quad n \in \mathbb{N} .
$$

Moreover,

$$
\operatorname{dim}_{F}\left(\mathcal{M}_{\tau}^{d}\right) \leq \log _{2}\left[N_{1 /(4 \Lambda)}(B(0,1 ; V), H)\right],
$$

where $N_{\varepsilon}(B(0,1 ; V), H)$ is the minimal number of balls of radius $\varepsilon$ in $H$ which are necessary to cover the unit ball of center 0 in $V$. Next, we define $\mathcal{M}_{\tau}$ by the formula (2.12). We conclude as in the proof of Theorem 2.5 that $\mathcal{M}_{\tau}$ is an exponential attractor for $S_{\tau}$ in $H$, with fractal dimension bounded by a constant independent of $\tau \in\left[\tau_{0}^{\prime}, \tau_{0}\right]$ and which attracts the bounded sets of $H$, uniformly with $\tau \in\left[\tau_{0}^{\prime}, \tau_{0}\right]$. This concludes the proof when Case 1 holds (the continuity holds only at $\tau=0$ ).

If Case 2 holds, we choose $r \geq 1 /\left(2 c_{1}^{\prime}\right)$ and we consider the set

$$
\mathcal{B}=\left\{w \in H:\|w\|_{V} \leq \hat{\rho}_{1} \text { and }\|w\|_{L^{a_{I}}} \leq \hat{\rho}_{I}^{\prime}\right\}
$$

which is absorbing in $H$, uniformly with respect to $\tau \in\left[0, \tau_{0}\right]$ since $\hat{\rho}_{1}>\rho_{1}$ and $\hat{\rho}_{I}^{\prime}>\rho_{I}^{\prime}$. We note that $a_{I}=I(2 p-2)+2 \geq I(2 p-2)$ and $a_{I} \geq 2 p$. Thus, assumptions (H1)-(H9) hold with $\beta=\gamma=1 / 2$ and, in (H6),

$$
\bar{\tau}_{0}(T)=\min \left\{\tau_{0}, 1 /\left(2 \tilde{h}_{2}\left(T,|\Omega|^{1 / s} \hat{\rho}_{I}^{\prime}\right)\right)\right\}>0,
$$

where $1 / s=1 /(I(2 p-2))-1 /(I(2 p-2)+2)$. The conclusions of Theorem 2.5 hold for some $\tau_{0}^{\prime} \in\left(0, \tau_{0}\right]$ small enough. For $\tau \in\left[\tau_{0}^{\prime}, \tau_{0}\right]$, we argue as previously. The proof is complete.

Corollary 6.2. For every $\tau \in\left[0, \tau_{0}\right]$, the semi-group $\left\{S_{\tau}(t), t \geq 0\right\}$ possesses a global attractor $\mathcal{A}_{\tau}$ in $H$ which is bounded in $V$ (uniformly in $\tau$ ), compact and connected in $H$. Moreover, $\operatorname{dist}_{H}\left\{\mathcal{A}_{\tau}, \mathcal{A}_{0}\right\} \rightarrow 0$ as $\tau \rightarrow 0^{+}$, and the fractal dimension of $\mathcal{A}_{\tau}$ is bounded by a constant independent of $\tau$.

Proof. Existence of a connected global attractor is a consequence of Theorem 1.1 in [30]. Upper semi-continuity of $\mathcal{A}_{\tau}$ as $\tau \rightarrow 0^{+}$is a consequence of assumptions (H1), 
(H5), (H7) and Proposition 1 in [31]. The upper bound on the fractal dimension follows from Theorem 6.1 and from the inclusion $\mathcal{A}_{\tau} \subset \mathcal{M}_{\tau}$.

Remark 6.3. Our upper bound on the fractal dimension of the attractors is quite crude (see (2.9), (6.1)). An upper bound which is optimal with respect to the physical parameters and which is based on the smoothing property has been obtained in [9] for a continuous-in-time reaction-diffusion system which includes equation (3.1). It is based on $L^{\infty}$-estimates (see also [8] for the classical construction based on the squeezing property). It could be interesting to investigate if such an optimal bound can be extended to our situation, where $L^{\infty}$-estimates can be derived for the continuous and the discretized problem.

\section{ACKNOWLEDGEMENTS}

The author is thankful to Alain Miranville for helpful discussions. The author also thanks the two anonymous referees for their useful comments.

\section{REFERENCES}

[1] M. Aida and A. Yagi. Global stability of approximation for exponential attractors. Funkcial. Ekvac., 47(2):251-276, 2004.

[2] S. Allen and J. Cahn. A microscopic theory for antiphase boundary motion and its application to antiphase domain coarsing. Acta. Metall., 27:1084-1095, 1979.

[3] A. V. Babin and M. I. Vishik. Attractors of evolution equations, volume 25 of Studies in Mathematics and its Applications. North-Holland Publishing Co., Amsterdam, 1992.

[4] T. Cazenave and A. Haraux. Introduction aux problèmes d'évolution semi-linéaires, volume 1 of Mathématiques \& Applications (Paris). Ellipses, Paris, 1990.

[5] N. Chafee and E. Infante. A bifurcation problem for a nonlinear partial differential equation of parabolic type. SIAM J. Appl. Anal., 4:17-37, 1974.

[6] V. V. Chepyzhov and M. I. Vishik. Attractors for equations of mathematical physics, volume 49 of American Mathematical Society Colloquium Publications. American Mathematical Society, Providence, RI, 2002.

[7] M. Coti Zelati and F. Tone. Multivalued attractors and their approximation: applications to the Navier-Stokes equations. Numer. Math., 122(3):421-441, 2012.

[8] A. Eden, C. Foias, B. Nicolaenko, and R. Temam. Exponential attractors for dissipative evolution equations, volume 37 of RAM: Research in Applied Mathematics. Masson, Paris; John Wiley \& Sons, Ltd., Chichester, 1994.

[9] M. Efendiev and A. Miranville. The dimension of the global attractor for dissipative reactiondiffusion systems. Appl. Math. Lett., 16(3):351-355, 2003.

[10] M. Efendiev, A. Miranville, and S. Zelik. Exponential attractors for a nonlinear reactiondiffusion system in $\mathbf{R}^{3}$. C. R. Acad. Sci. Paris Sér. I Math., 330(8):713-718, 2000.

[11] M. Efendiev, A. Miranville, and S. Zelik. Exponential attractors for a singularly perturbed Cahn-Hilliard system. Math. Nachr., 272:11-31, 2004.

[12] M. Efendiev and A. Yagi. Continuous dependence on a parameter of exponential attractors for chemotaxis-growth system. J. Math. Soc. Japan, 57(1):167-181, 2005.

[13] E. Ezzoug, O. Goubet, and E. Zahrouni. Semi-discrete weakly damped nonlinear 2-D Schrödinger equation. Differential Integral Equations, 23(3-4):237-252, 2010.

[14] P. Fabrie, C. Galusinski, and A. Miranville. Uniform inertial sets for damped wave equations. Discrete Contin. Dynam. Systems, 6(2):393-418, 2000.

[15] P. Fabrie, C. Galusinski, A. Miranville, and S. Zelik. Uniform exponential attractors for a singularly perturbed damped wave equation. Discrete Contin. Dyn. Syst., 10(1-2):211-238, 2004.

[16] C. Galusinski. Perturbations singulières de problèmes dissipatifs : étude dynamique via l'existence et la continuité d'attracteurs exponentiels. PhD thesis, Université de Bordeaux, 1996.

[17] S. Gatti, M. Grasselli, A. Miranville, and V. Pata. A construction of a robust family of exponential attractors. Proc. Amer. Math. Soc., 134(1):117-127 (electronic), 2006. 
[18] D. Gilbarg and N. S. Trudinger. Elliptic partial differential equations of second order. Classics in Mathematics. Springer-Verlag, Berlin, 2001.

[19] J. K. Hale. Asymptotic behavior of dissipative systems, volume 25 of Mathematical Surveys and Monographs. American Mathematical Society, Providence, RI, 1988.

[20] D. Henry. Geometric theory of semilinear parabolic equations, volume 840 of Lecture Notes in Mathematics. Springer-Verlag, Berlin-New York, 1981.

[21] O. Ladyzhenskaya. Attractors for semigroups and evolution equations. Lezioni Lincee. Cambridge University Press, Cambridge, 1991.

[22] J.-L. Lions. Quelques méthodes de résolution des problèmes aux limites non linéaires. Dunod; Gauthier-Villars, Paris, 1969.

[23] M. Marion. Attractors for reaction-diffusion equations: existence and estimate of their dimension. Appl. Anal., 25(1-2):101-147, 1987.

[24] A. Miranville and S. Zelik. Attractors for dissipative partial differential equations in bounded and unbounded domains. In Handbook of differential equations: evolutionary equations. Vol. IV, Handb. Differ. Equ., pages 103-200. Elsevier/North-Holland, Amsterdam, 2008.

[25] G. Raugel. Global attractors in partial differential equations. In Handbook of dynamical systems, Vol. 2, pages 885-982. North-Holland, Amsterdam, 2002.

[26] G. R. Sell and Y. You. Dynamics of evolutionary equations, volume 143 of Applied Mathematical Sciences. Springer-Verlag, New York, 2002.

[27] J. Shen. Convergence of approximate attractors for a fully discrete system for reaction-diffusion equations. Numer. Funct. Anal. Optim., 10(11-12):1213-1234 (1990), 1989.

[28] J. Shen. Long time stability and convergence for fully discrete nonlinear Galerkin methods. Appl. Anal., 38(4):201-229, 1990.

[29] A. M. Stuart and A. R. Humphries. Dynamical systems and numerical analysis, volume 2 of Cambridge Monographs on Applied and Computational Mathematics. Cambridge University Press, Cambridge, 1996.

[30] R. Temam. Infinite-dimensional dynamical systems in mechanics and physics, volume 68 of Applied Mathematical Sciences. Springer-Verlag, New York, second edition, 1997.

[31] X. Wang. Approximation of stationary statistical properties of dissipative dynamical systems: time discretization. Math. Comp., 79(269):259-280, 2010.

[32] X. Wang. Numerical algorithms for stationary statistical properties of dissipative dynamical systems. Discrete Contin. Dyn. Syst., 36(8):4599-4618, 2016.

Laboratoire de Mathématiques et Applications, Université de Poitiers, CNRS, F86962 Chasseneuil, France. EMAIL: Pierre@math. Univ-Poititers.Fr 